ESAIM: COCV 27 (2021) S16

https://doi.org/10.1051/cocv/2020067
ESAIM: Control, Optimisation and Calculus of Variations

www.esaim-cocv.org

\title{
A HIERARCHY OF MULTILAYERED PLATE MODELS
}

\author{
Miguel de Benito Delgado and Bernd Schmidt*
}

\begin{abstract}
We derive a hierarchy of plate theories for heterogeneous multilayers from three dimensional nonlinear elasticity by means of $\Gamma$-convergence. We allow for layers composed of different materials whose constitutive assumptions may vary significantly in the small film direction and which also may have a (small) pre-stress. By computing the $\Gamma$-limits in the energy regimes in which the scaling of the pre-stress is non-trivial, we arrive at linearised Kirchhoff, von Kármán, and fully linear plate theories, respectively, which contain an additional spontaneous curvature tensor. The effective (homogenised) elastic constants of the plates will turn out to be given in terms of the moments of the pointwise elastic constants of the materials.
\end{abstract}

Mathematics Subject Classification. 74K20, 49J45, 74G65.

Received June 4, 2019. Accepted October 6, 2020.

\section{INTRODUCTION}

The derivation of effective theories for thin structures such as beams, rods, plates and shells is a classical problem in continuum mechanics. Fundamental results in formulating adequate dimensionally reduced theories for three-dimensional elastic objects have already been obtained by Euler [18], Kirchhoff [30] and von Kármán [55], cf. also [9, 10, 37].

A physical plate, given by a domain $\Omega_{h}=\omega \times(-h / 2, h / 2) \subset \mathbb{R}^{3}$, is identified with a hyperelastic body of height $h$ "much smaller" than the lengths of the sides of $\omega$. The plane domain $\omega \subset \mathbb{R}^{2}$ constitutes the mid-layer of the plate. We assume that the body has a (possibly non-homogeneous) stored energy density $W$ (precise conditions on $W$ will be specified later) and, after deformation by $\tilde{y}: \Omega_{h} \rightarrow \mathbb{R}^{3}$, the total elastic energy

$$
E_{h}(\tilde{y})=\int_{\Omega_{h}} W(z, \nabla \tilde{y}(z)) \mathrm{d} z .
$$

The problem amounts to identifying effective functionals in the limit $h \rightarrow 0$ operating on dimensionally reduced deformations of the mid-plane. In spite of its long history, rigorous results in this direction relating classical models for plates to the parent three-dimensional elasticity theory have only been obtained comparatively recently.

In order to avoid working on a changing domain, a rescaling $x_{3}=z_{3} / h$ is performed to obtain a fixed $\Omega_{1}$. We set $z_{h}\left(x_{1}, x_{2}, x_{3}\right)=\left(x_{1}, x_{2}, h x_{3}\right)$ and we consider instead of a deformation $\tilde{y}: \Omega_{h} \rightarrow \mathbb{R}^{3}$, the rescaled one

Keywords and phrases: Multilayers, dimension reduction, effective plate theories, Gamma-convergence.

Universität Augsburg, Augsburg, Germany.

* Corresponding author: bernd.schmidt@math.uni-augsburg.de 
$y_{h}: \Omega_{1} \rightarrow \mathbb{R}^{3}, y_{h}(x)=\tilde{y}\left(z_{h}(x)\right)$. We define the energy per unit volume as $J_{h}=\frac{1}{h} E_{h}$, which after a change of variables becomes

$$
J_{h}(y)=\int_{\Omega_{1}} W\left(x, \nabla_{h} y(x)\right) \mathrm{d} x
$$

where $\nabla_{h}=\left(\partial_{1}, \partial_{2}, \partial_{3} / h\right)$.

After first results in linear elasticity had been established, see $[2,4]$, perhaps the first work to derive a nonlinearly elastic, lower dimensional theory with a rigorous analysis using variational convergence was [3] for the case of strings. In the context of nonlinear plates we consider the rescaled functionals

$$
J_{h}^{\beta}(y)=\frac{1}{h^{\beta}} \int_{\Omega_{1}} W\left(x, \nabla_{h} y(x)\right) \mathrm{d} x .
$$

Inspired by the work in [3], a non-linear membrane theory is derived in [33] for $\beta=0$. The range $\beta \in(0,2)$ is the so-called constrained membrane regime, which still is not fully explored, except under certain kinds of boundary conditions or assumed admissible deformations, see, e.g., [5] and the work in [13]. For minimizers under body forces, convergence results have been obtained in [11].

Most significant in view of our setup are the contributions to the cases $\beta \geq 2$. In [22] Friesecke, James and Müller prove the fundamental geometric rigidity estimate which carries Korn's inequality to the nonlinear setting and utilise it to obtain the non-linear Kirchhoff theory of pure bending under an isometry constraint in the case $\beta=2$. This estimate is at the core of most of the later developments in this area. In their seminal paper [23], the same authors exploit the quantitative geometric rigidity estimate of [22] in a systematic investigation of limits for the whole range of scalings $\beta \in[2, \infty)$, deriving the first hierarchy of limit models. They also provide a thorough (albeit succinct) overview of the state of the art around 2006. The lecture ([41], Chap. 2) provides a nice walkthrough of this paper, as well as abundant references and open problems as of 2017 .

This variational approach has been extended and revisited in a variety of different contexts, among them more complex shell geometries [21, 36], more basic atomistic models [8, 50], or more complicated material properties as incompressibility [12], brittleness [53] or oscillatory dependence on the space variable [27, 28, 44]. Moreover, the convergence of equilibria [40,43] and even dynamic solutions [1] have been established.

The focus in this contribution is on materials whose reference configuration is subjected to stresses (one speaks of pre-strained or pre-stressed bodies) and whose energy density exhibits a dependence on the out-of-plane direction (modelling multilayered plates). Examples of these situations are heated materials, crystallisations on top of a substrate and multilayered plates.

For $\beta=2$ the second author derived in $[51,52]$ an effective Kirchhoff theory for stored energy densities of the form $W\left(x_{3}, F\right)=W_{0}\left(x_{3}, F\left(I+h B^{h}\left(x_{3}\right)\right)\right)$, depending explicitly on the out-of-plane coordinate $x_{3}$ and a "mismatch tensor" $B^{h}\left(x_{3}\right)$ which measures the deviation of the energy well argmin $W\left(x_{3}, \cdot\right)$ from the rigid motions argmin $W_{0}\left(x_{3}, \cdot\right)=\mathrm{SO}(3)$. We remark that the regime $\beta=2$ is precisely adapted to capture the effects of a misfit $h B^{h}$ scaling linearly in $h$. In the simplest case with linearly changing $B^{h}\left(x_{3}\right)=a x_{3} I$ one obtains a $\Gamma$-limit $I_{\mathrm{Ki}}$ with

$$
I_{\mathrm{Ki}}(y)=\frac{1}{24} \int_{\omega} Q\left(\mathrm{II}-a_{1} I\right)-a_{2} \mathrm{~d} x
$$

if $y \in \mathcal{A}$ (and $I_{\mathrm{Ki}}(y)=+\infty$ if not), where $\mathcal{A}$ is a suitable class of admissible deformations (isometric immersions). $Q$ is a quadratic form acting on the shape tensor II (the second fundamental form of $y$ ). The coefficients of $Q$ and the numbers $a_{1}, a_{2}$ can be explicitly computed. In [51,52] also a thorough investigation of the shape of energy minimisers (for free boundary conditions) is provided which shows that the optimal configurations are rolled-up portions of cylinders whose winding directions and radii are determined by the material parameters and the misfit tensor. 
The main goal of our work is to extend such an analysis to the energy regimes $\beta>2$. This will in particular allow for a more subtle investigation of the effect of general pre-strain scalings of the form $h^{\alpha-1} B^{h}\left(x_{3}\right), \alpha>2$. Indeed, as we will see, such pre-strains will result in contributions to the limiting functional which are both finite and non-trivial precisely if $\beta=2 \alpha-2$. A main source of motivation are physical experiments which show that there are situations in which optimal configurations are spherical caps (paraboloids with positive Gauß curvature) rather than cylinders, $[17,19,20,29,39,49]$. We will see that indeed this discrepancy can be explained in terms of different energy scaling regimes, where the von Kármán scaling $\beta=4$ is critical. In the present paper we lay the foundation for this by deriving effective plate theories for pre-strained multilayers. We analyse the functionals obtained here in depth in our companion paper [15].

From a more general perspective, a thorough theoretical understanding of multilayers is not only interesting from a mathematical point of view. Such structures are of great interest in engineering applications, see [48]. In particular, the mechanism through which a pre-strain provokes mechanical displacement has been of great interest: It allows to access and manipulate objects even at the nanoscale in a convenient and feasible way, see, e.g., $[24,45,47,54]$, where a misfit of the equilibria of the constituents is used to achieve a self-organised fabrication of nano-scrolls. On a macroscopic scale one also observes rather complicated material behaviour that is caused by pre-strains which induce a non-Euclidean target metric, see e.g. [31]. In [17] recent experiments are reported on elastomer polydimethylsiloxane bilayers which fold into complex three dimensional configurations in response to programmed misfit distributions.

In fact, the derivation of effective models for thin plates with a pre-strain has received a lot of attention in recent contributions. With no attempt to be exhaustive we mention [6, 16, 32, 34, 35, 38, 51, 52]. Yet, as we are primarily interested in modeling multilayers, our set-up is quite different from those contributions, where the pre-strain and elastic moduli depend only on the in-plane variables. In particular, in [35] the authors derive the von Kármán functional with a spontaneous curvature term for pre-stressed plates in the case $\beta=4$. The same functional also arises in a special case of our setting, $c f$. (1.1) with $\theta=1$ below. However, their setup is not comparable to our situation. On the one hand, it is even more general as an explicit $\left(x_{1}, x_{2}\right)$ dependence of the misfit is allowed. On the other hand, there is no explicit $x_{3}$ dependence, neither on the pre-strain nor on the material properties, as would be necessary to model multilayers. As discussed above, in the bending dominated regime, models in which both pre-strain and material parameters were allowed to vary in the thin film direction were already discussed in [51, 52]. Generalizing [35, 52], the recent paper [34] considers a variety of scaling regimes also for thickness dependent pre-strains. However, our treatment of energy densities which may vary considerably in the thin film direction is, as we will see, quite subtle. A main source of technical difficulties is the fact that in our situation we can no longer expect the mid plane to follow the limiting plate deformation exactly. This phenomenon can be observed already in the simplest situation of a bilayer with one layer being much softer than the other. If rolled up, the unstretched plane will move into the stiffer layer, to an extent which depends on the local curvature.

From a technical point of view, it turns out that in our setup the von Kármán case $\beta=4$ is in fact a rather straightforward extension of $[23,52]$. The regime $\beta>4$ is however a bit more involved. In contrast to the homogeneous case in [23], the dependence of the resulting limiting theory on the in-plane displacements may be non-trivial so it cannot be discarded by setting it to 0 without loss of generality. The scaling in the linearised Kirchhoff case $\beta \in(2,4)$ turns out to be the most difficult. In order to construct recovery sequences we need to provide a representation result for symmetric tensor fields on $\omega$ in terms of symmetrised gradients and solutions to the non-elliptic Monge-Ampere equation $\operatorname{det} \nabla^{2} v=0, c f$. Theorem 7.3.

From a modelling point of view, a main novelty is in our introducing a new interpolating regime in between the linearised Kirchhoff case $\beta<4$ and the fully linear case $\beta>4$. This is motivated by our findings in [15] which show that minimisers (after rescaling) coincide for all $\beta \in(2,4)$ (parts of a cylinder) and for all $\beta \in(4, \infty$ ) (parts of a parabolic cap). In this sense, the exponent $\beta=4$ in the von Kármán case is critical. We introduce an additional fine scale inducing a new scaling regime $\theta h^{4}$ with $\theta \in(0, \infty)$ and obtain von Kármán functionals that upon varying $\theta$ continuously connect the extreme cases $\theta \rightarrow 0$ and $\theta \rightarrow \infty$, which turn out to reduce to the functionals obtained for $\beta>4$ and $\beta<4$, respectively. In the simplest non-trivial example, the prototypical 
limit functional is of von Kármán type:

$$
\mathcal{I}_{\mathrm{vK}}^{\theta}(u, v)=\frac{\theta}{2} \int_{\omega} Q_{2}\left(\nabla_{s} u+\frac{1}{2} \nabla v \otimes \nabla v\right) \mathrm{d} x+\frac{1}{24} \int_{\omega} Q_{2}\left(\nabla^{2} v-I\right) \mathrm{d} x
$$

In contrast to the cases $\beta \neq 4$ minimisers of this functional are not explicit. We discuss their behaviour in detail in [15], in particular, how they interpolate in between $\beta<4$ and $\beta>4$.

With a view to the applicability of our results in engineering problems, we emphasize that our effective models are completely explicit. The effective elastic moduli and spontaneous curvature terms in the limiting plate theory are explicitly computable from the elastic moduli and pre-strain of the constituents of the multilayer. Explicit formulae identifying the limiting homogenised material constants in terms of the moments in $x_{3}$ of the corresponding parameters within the individual layers are provided in our companion paper [15]. There, we also discuss a BGaAs/InGaAs bilayer in order to give a concrete and explicit example which connects our theory to experimental results.

\section{Outline}

Having fixed the precise setup in Section 2, in Section 3 we present our main results: Theorem 3.1 on $\Gamma$ convergence in a hierarchy of energy scalings and Theorem 3.3 on the asymptotic behaviour of the interpolating von Kármán functional for $\theta \rightarrow 0$ or $\theta \rightarrow \infty$. We then recall some basic results on compactness and explicit representations for the limit strains from [23] in Section 4. Proofs of lower and upper bounds in Theorem 3.1 are collected in Section 5, where we obtain (1.1) and more general functionals. In Section 6 we show how the von Kármán functional interpolates between different theories. Finally, in Section 7 we prove some density and matrix representation theorems essential for the construction of recovery sequences and identification of minimisers in the linearised Kirchhoff regime.

\section{Notation}

We denote by $e_{1}, e_{2}, e_{3}$ the standard basis vectors in $\mathbb{R}^{3}$ and write $x=\left(x^{\prime}, x_{3}\right) \in \mathbb{R}^{3}, x^{\prime} \in \mathbb{R}^{2}$. The spaces of symmetric and antisymmetric $n \times n$ matrices are $\mathbb{R}_{\mathrm{sym}}^{n \times n}$ and $\mathbb{R}_{\text {ant }}^{n \times n}$, respectively. $A_{\mathrm{sym}}=\operatorname{sym} A=\frac{1}{2}\left(A+A^{\top}\right)$ is the symmetric part and $A_{\text {ant }}=\operatorname{ant} A=\frac{1}{2}\left(A-A^{\top}\right)$ the antisymmetric part of a square matrix $A$.

Attaching a row and a column of zeros to a matrix $G \in \mathbb{R}^{2 \times 2}$ leads to $\hat{G}:=\sum_{\alpha, \beta=1}^{2} G_{\alpha \beta} e_{\alpha} \otimes e_{\beta} \in \mathbb{R}^{3 \times 3}$, conversely, $\check{B} \in \mathbb{R}^{2 \times 2}$ is the matrix resulting from the deletion of the third row and column of any $B \in \mathbb{R}^{3 \times 3}$. If $Q(\cdot)$ is a quadratic form, we denote the associated bilinear form by $Q[\cdot, \cdot]$.

For a scalar function $f: \mathbb{R}^{3} \rightarrow \mathbb{R}, \nabla f=\left(\partial_{1} f, \partial_{2} f, \partial_{3} f\right)^{\top}$ is a column vector, whereas for $y: \mathbb{R}^{3} \rightarrow \mathbb{R}^{3}$ we have $\nabla y \in \mathbb{R}^{3 \times 3}$ with rows $\nabla^{\top} y_{i}$, i.e., $(\nabla y)_{i j}=y_{i, j}=\partial_{j} y_{i}, i, j \in\{1,2,3\}$. Its left $3 \times 2$ submatrix is $\nabla^{\prime} y$, its rescaled gradient $\nabla_{h} y=\left(\partial_{1} y, \partial_{2} y, 1 / h \partial_{3} y\right)$. Moreover, $\nabla_{s} u=\frac{1}{2}\left(\nabla u+\nabla^{\top} u\right)$, is the symmetrised gradient of $u: \mathbb{R}^{2} \rightarrow \mathbb{R}^{2}, \nabla^{2} v$ the Hessian matrix of $v: \mathbb{R}^{n} \rightarrow \mathbb{R}$.

Let $\omega \subset \mathbb{R}^{2}$. We set $\hat{\nabla} v:=\left(\partial_{1} v, \partial_{2} v, 0\right)^{\top} \in \mathbb{R}^{3}$ for $v: \omega \rightarrow \mathbb{R}, \hat{\nabla} u:=\sum_{\alpha, \beta=1}^{2}\left(\nabla^{\prime} u\right)_{\alpha \beta} e_{\alpha} \otimes e_{\beta} \in \mathbb{R}^{3 \times 3}$ for $u: \omega \rightarrow \mathbb{R}^{2}$ and $\hat{\nabla} b:=\sum_{\alpha=1}^{3} \sum_{\beta=1}^{2}\left(\nabla^{\prime} b\right)_{\alpha \beta} e_{\alpha} \otimes e_{\beta} \in \mathbb{R}^{3 \times 3}$ for $b: \omega \rightarrow \mathbb{R}^{3}$.

The norm on Sobolev spaces is $\|\cdot\|_{k, p, \Omega}=\|\cdot\|_{W^{k, p}(\Omega)}$ and, in particular, $\|\cdot\|_{0, p, \Omega}$ is the $L^{p}$-norm. We will omit the domain when it is clear from the context.

We abbreviate $A_{\theta}:=\nabla_{s} u_{\theta}+\frac{1}{2} \nabla v_{\theta} \otimes \nabla v_{\theta}$, mostly in Section 6 and set $(f)_{\omega}:=\frac{1}{|\omega|} \int_{\omega} f\left(x^{\prime}\right) \mathrm{d} x^{\prime}$ is the average of $f$ over $\omega$. 


\section{The SETTING}

As described in Section 1, we consider a sequence of increasingly thin domains $\Omega_{h}:=\omega \times(-h / 2, h / 2) \subset \mathbb{R}^{3}$ and rescale them to

$$
\Omega_{1}:=\omega \times(-1 / 2,1 / 2) \subset \mathbb{R}^{3},
$$

where $\omega \subset \mathbb{R}^{2}$ is a bounded domain with Lipschitz boundary. As a consequence of the rescaling, instead of maps $\tilde{y}: \Omega_{h} \rightarrow \mathbb{R}^{3}$, we consider the rescaled deformations

$$
y: \Omega_{1} \rightarrow \mathbb{R}^{3}, \quad x \mapsto y(x)=\tilde{y}\left(x_{1}, x_{2}, h x_{3}\right),
$$

belonging to the space $Y:=W^{1,2}\left(\Omega_{1} ; \mathbb{R}^{3}\right)$. For each scaling ${ }^{1} \alpha \in(2, \infty)$, and for all deformations $y \in Y$, define the scaled elastic energy per unit volume:

$$
\mathcal{I}_{\alpha}^{h}(y)=\frac{1}{h^{2 \alpha-2}} \int_{\Omega_{1}} W_{\alpha}^{h}\left(x_{3}, \nabla_{h} y(x)\right) \mathrm{d} x,
$$

where $\nabla_{h}=\left(\partial_{1}, \partial_{2}, \partial_{3} / h\right)^{\top}$ is the gradient operator resulting after the change of coordinates described in Section 1. For the sake of conciseness, we will present most results below for all scalings simultaneously, adding the parameter $\alpha$ to much of the notation. The energy density for $\alpha \neq 3$ is given by

$$
W_{\alpha}^{h}\left(x_{3}, F\right)=W_{0}\left(x_{3}, F\left(I+h^{\alpha-1} B^{h}\left(x_{3}\right)\right)\right), \quad F \in \mathbb{R}^{3 \times 3},
$$

where $B^{h}:(-1 / 2,1 / 2) \rightarrow \mathbb{R}^{3 \times 3}$ describes the internal misfit and $W_{0}$ the stored energy density of the reference configuration. In the regime $\alpha=3$ we include an additional parameter $\theta>0$ controlling further the amount of misfit in the model:

$$
W_{\alpha=3}^{h}\left(x_{3}, F\right)=W_{0}\left(x_{3}, F\left(I+h^{2} \sqrt{\theta} B^{h}\left(x_{3}\right)\right)\right), \quad F \in \mathbb{R}^{3 \times 3},
$$

and we later write $\tilde{B}^{h}=\sqrt{\theta} B^{h}$. Note that given the choice $h^{\alpha-1}$ for the scaling of the misfit, the fact that in the limit it will be again scaled quadratically entails our choice of rescaling the energy with $h^{-2(\alpha-1)}$. Indeed on coarser energy scales $h^{\beta}, \beta<2(\alpha-1)$, such a misfit will not contribute to the limiting functional. (This case is indeed also covered in our analysis below by considering $\mathcal{I}_{1+\beta / 2}^{h}$ with $B^{h} \rightarrow 0$.) On the other hand, on finer energy scales $h^{\beta}, \beta>2(\alpha-1)$, the pre-strain will typically lead to diverging energy terms in the limit (see (2.5), (2.6) and the computation of the lower bounds in Thm. 5.1). Our assumptions for $B^{h}$ and $W_{0}$ are those of ([52], Asm. 1.1):

\section{Assumption 2.1.}

(a) For a.e. $t \in(-1 / 2,1 / 2), W_{0}(t, \cdot)$ is continuous on $\mathbb{R}^{3 \times 3}$ and $C^{2}$ in a neighbourhood of $\mathrm{SO}(3)$ which does not depend on $t$.

(b) The map $t \mapsto Q_{3}(t, \cdot)=D^{2} W_{0}(t, I)[\cdot, \cdot]$ is in $L^{\infty}\left((-1 / 2,1 / 2) ; \mathbb{R}^{9 \times 9}\right)$.

(c) The map

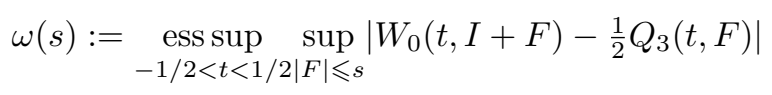

shall satisfy $\omega(s)=o\left(s^{2}\right)$ as $s \rightarrow 0$.

\footnotetext{
${ }^{\mathrm{I}}$ In the notation of Section 1 we have $\beta=2 \alpha-2$.
} 
(d) For all $F \in \mathbb{R}^{3 \times 3}$ and all $R \in \mathrm{SO}(3)$

$$
W_{0}(t, F)=W_{0}(t, R F) .
$$

(e) For a.e. $t \in(-1 / 2,1 / 2), W_{0}(t, F)=0$ if $F \in \mathrm{SO}(3)$ and

$$
\underset{-1 / 2<t<1 / 2}{\operatorname{ess} i n f} W_{0}(t, F) \geqslant c \operatorname{dist}^{2}(F, \mathrm{SO}(3)),
$$

for all $F \in \mathbb{R}^{3 \times 3}$ and some $c>0$.

(f) $B^{h} \rightarrow B$ in $L^{\infty}\left((-1 / 2,1 / 2) ; \mathbb{R}^{3 \times 3}\right)$.

The Hessian

$$
Q_{3}(t, F):=D^{2} W_{0}(t, I)[F, F]=\frac{\partial^{2} W_{0}(t, I)}{\partial F_{i j} \partial F_{i j}} F_{i j} F_{i j},
$$

for $t \in(-1 / 2,1 / 2), F \in \mathbb{R}^{3 \times 3}$ is twice the quadratic form of linear elasticity theory, which results after a linearisation of $W_{0}$ around the identity. By Assumption 2.1.e it is positive definite on symmetric matrices and vanishing on antisymmetric matrices. We note in passing two consequences of the above conditions. First, frame invariance (Assumption 2.1.d) extends to the second derivative where defined, i.e.

$$
D^{2} W_{0}(t, R)[F R, F R]=D^{2} W_{0}(t, I)[F, F]=Q_{3}(t, F) .
$$

Second, the energy $W_{0}$ grows at most quadratically in a neighbourhood of $\mathrm{SO}(3)$, i.e., there exists a $C>0$ such that for sufficiently small $|F|$ it holds that for all $t \in(-1 / 2,1 / 2)$ :

$$
W_{0}(t, I+F) \leqslant C \operatorname{dist}^{2}(I+F, \mathrm{SO}(3))
$$

Define $Q_{2}$ to be the quadratic form on $\mathbb{R}^{2 \times 2}$ obtained by relaxation of $Q_{3}$ among stretches in the $x_{3}$ direction:

$$
Q_{2}(t, G):=\min _{c \in \mathbb{R}^{3}} Q_{3}\left(t, \hat{G}+c \otimes e_{3}\right), \text { for } t \in(-1 / 2,1 / 2), G \in \mathbb{R}^{2 \times 2},
$$

where $e_{3}=(0,0,1) \in \mathbb{R}^{3}$. (See the last paragraph of Sect. 1 for the definition of $\hat{G}$.) This process effectively minimises away the effect of transversal strain. Solving the minimisation problem yields a map $\mathcal{L}:(-1 / 2,1 / 2) \times$ $\mathbb{R}^{2 \times 2} \rightarrow \mathbb{R}^{3}$, linear in its second argument, which attains the minimum:

$$
Q_{2}(t, G)=Q_{3}\left(t, \hat{G}+\mathcal{L}(t, G) \otimes e_{3}\right)
$$

In particular, also the $Q_{2}(t, \cdot)$ are positive definite on symmetric matrices and vanishing on antisymmetric matrices. In fact, by Assumptions 2.1.b and 2.1.e we have the bounds

$$
Q_{2}(t, F) \gtrsim|F|^{2} \quad \forall F \in \mathbb{R}_{\mathrm{sym}}^{2 \times 2} \quad \text { and } \quad|\mathcal{L}(t, F)| \lesssim|F| \forall F \in \mathbb{R}^{2 \times 2},
$$

uniformly in $t \in(-1 / 2,1 / 2)$.

For the regimes $\alpha \geqslant 3$, we define the effective form

$$
\bar{Q}_{2}(E, F):=\int_{-1 / 2}^{1 / 2} Q_{2}(t, E+t F+\check{B}(t)) \mathrm{d} t
$$


with $E, F \in \mathbb{R}^{2 \times 2}$ (see the last paragraph of Sect. 1 for the definition of $\check{B}$ ). For $\alpha \in(2,3)$ we consider its relaxation

$$
\bar{Q}_{2}^{\star}(F):=\min _{E \in \mathbb{R}^{2 \times 2}} \bar{Q}_{2}(E, F)=\min _{E \in \mathbb{R}_{\mathrm{sym}}^{2 \times 2}} \int_{-1 / 2}^{1 / 2} Q_{2}(t, E+t F+\check{B}(t)) \mathrm{d} t .
$$

For the case $\alpha=3$, we include an additional parameter $\theta>0$ as discussed in page 5 and later write $\tilde{B}=\sqrt{\theta} B$. Both $\bar{Q}_{2}$ and $\bar{Q}_{2}^{\star}$ are non-negative quadratic polynomials. Solving the quadratic minimization problems in $(2.2)$ and (2.6) and computing the $t$-integrations in (2.5) and (2.6) one obtains explicit formulae for their coefficients in terms the first moments

$$
\int_{-1 / 2}^{1 / 2} t^{k} Q_{3}(t, \cdot) \mathrm{d} t, \quad \int_{-1 / 2}^{1 / 2} t^{k} B(t) \mathrm{d} t
$$

$k=0,1,2$, in the small film direction of the elastic moduli and the misfit tensor, respectively. This is made explicit in Section 2.2 of our companion paper [15].

For fixed $\alpha \in(2, \infty)$ we say that a sequence $\left(y^{h}\right)_{h>0} \subset Y$ has finite scaled energy if there exists some constant $C>0$ such that

$$
\operatorname{lsup}_{h \rightarrow 0} \mathcal{I}_{\alpha}^{h}\left(y^{h}\right) \leqslant C
$$

After some corrections we will have precompactness of such sequences, thus essentially proving that the family $\mathcal{I}_{\alpha}^{h}$ is equicoercive, the essential condition for the fundamental theorem of $\Gamma$-convergence showing convergence of minimisers and energies. This compactness takes place in adequate target ambient spaces

$$
X_{\alpha}= \begin{cases}W^{1,2}(\omega ; \mathbb{R}) & \text { if } \alpha \in(2,3), \\ W^{1,2}\left(\omega ; \mathbb{R}^{2}\right) \times W^{1,2}(\omega ; \mathbb{R}) & \text { if } \alpha \geqslant 3\end{cases}
$$

equipped with the weak topology.

An essential ingredient in arguments with $\Gamma$-convergence is the choice of sequential convergence to obtain (pre-)compactness. For the lower bounds we may suppose that a sequence $\left(y^{h}\right)_{h>0}$ has finite scaled energy, which enables Lemma 4.1 for the identification of the limits, $c f$. Section 4. This requires us to work with the corrected deformations $\rho\left(y^{h}\right):=\left(\bar{R}^{h}\right)^{\top} y^{h}-\bar{c}^{h}$, for some constants $\bar{R}^{h} \in \mathrm{SO}(3)$ and $\bar{c}^{h} \in \mathbb{R}^{3}$ depending on $y^{h}$, see (4.1). ${ }^{2}$ We choose to encode this transformation into the definition of $\Gamma$-convergence via maps $P_{\alpha}^{h}$ (Def. 2.3) for general transformations $\rho$ with arbitrary $R^{h} \in \mathrm{SO}(3)$ and $c^{h} \in \mathbb{R}^{3}$. Despite adding clutter to the notation, this helps to highlight and isolate the technical requirement of the sequences involved with special rigid transformations. ${ }^{3}$

Definition 2.2. Let $Y:=W^{1,2}\left(\Omega_{1} ; \mathbb{R}^{3}\right)$ and $X_{\alpha}$ as in (2.7). We say that a sequence $\left(y^{h}\right)_{h>0} \subset Y P^{h}$-converges to some $w \in X_{\alpha}$ if and only if there exist constants $R^{h} \in \mathrm{SO}(3), c^{h} \in \mathbb{R}^{3}$ which define maps

$$
\rho: Y \rightarrow Y, \quad y^{h} \mapsto \rho\left(y^{h}\right):=\left(R^{h}\right)^{\top} y^{h}-c^{h},
$$

such that

$$
P_{\alpha}^{h}\left(y^{h}\right) \rightarrow w \quad \text { weakly in } X_{\alpha}
$$

\footnotetext{
${ }^{2}$ These maps "remove" rigid movements from the $y^{h}$ bringing them close to the identity. Note that the energy is not affected by this change because of frame invariance (Assumption 2.1.d).

${ }^{3}$ We only require that there be some constants $R^{h}, c^{h}$ for $P^{h}$-convergence. In order to obtain compactness and in the lower bounds we will take the specific ones given in Lemma 4.1 whereas for the recovery sequences we will use $R^{h}=I, c^{h}=0$.
} 
where

$$
P_{\alpha}^{h}: Y \rightarrow X_{\alpha}, y^{h} \mapsto \begin{cases}v_{\alpha}^{h}, & \text { if } \alpha \in(2,3), \\ \left(u_{\theta}^{h}, v_{\theta}^{h}\right) & \text { if } \alpha=3, \\ \left(u_{\alpha}^{h}, v_{\alpha}^{h}\right), & \text { if } \alpha>3,\end{cases}
$$

and we defined:

For $\alpha \neq 3$ and $x^{\prime} \in \omega$, the scaled, averaged and corrected in-plane and out-of-plane displacements:

$$
\left\{\begin{array}{l}
u_{\alpha}^{h}\left(x^{\prime}\right):=\frac{1}{h^{\gamma}} \int_{-1 / 2}^{1 / 2}\left(\rho\left(y^{h}\right)^{\prime}\left(x^{\prime}, x_{3}\right)-x^{\prime}\right) \mathrm{d} x_{3}, \\
v_{\alpha}^{h}\left(x^{\prime}\right):=\frac{1}{h^{\alpha-2}} \int_{-1 / 2}^{1 / 2} \rho\left(y^{h}\right)_{3}\left(x^{\prime}, x_{3}\right) \mathrm{d} x_{3},
\end{array}\right.
$$

where $\rho\left(y^{h}\right)^{\prime}\left(x^{\prime}, x_{3}\right)=\left(\rho\left(y^{h}\right)_{1}, \rho\left(y^{h}\right)_{2}\right)\left(x^{\prime}, x_{3}\right)$ and

$$
\gamma=\left\{\begin{array}{rll}
2(\alpha-2) & \text { if } & \alpha \in(2,3) \\
\alpha-1 & \text { if } & \alpha>3
\end{array}\right.
$$

For $\alpha=3$ and $x^{\prime} \in \omega$, introducing the additional parameter $\theta>0$ :

$$
\left\{\begin{aligned}
u_{\theta}^{h}\left(x^{\prime}\right) & :=\frac{1}{\theta h^{2}} \int_{-1 / 2}^{1 / 2}\left[\rho\left(y^{h}\right)^{\prime}\left(x^{\prime}, x_{3}\right)-x^{\prime}\right] \mathrm{d} x_{3} \\
v_{\theta}^{h}\left(x^{\prime}\right) & :=\frac{1}{\sqrt{\theta} h} \int_{-1 / 2}^{1 / 2} \rho\left(y^{h}\right)_{3}\left(x^{\prime}, x_{3}\right) \mathrm{d} x_{3} .
\end{aligned}\right.
$$

For $\alpha=3$, we overload the notation with the parameter $\theta$ writing $\left(u_{\theta}^{h}, v_{\theta}^{h}\right)$ and $P_{\theta}^{h}$ instead of $\left(u_{\alpha}^{h}, v_{\alpha}^{h}\right)$ or $P_{\alpha}^{h}$, letting the letter used in the subindex resolve ambiguity.

With Definition 2.2 we can specify precisely what we mean by $\Gamma$-convergence of the energies $(2.1):^{4}$

Definition 2.3. Let $\alpha>2$. We say that the family of scaled elastic energies $\left\{\mathcal{I}_{\alpha}^{h}: Y \rightarrow \mathbb{R}\right\}_{h>0}, h>0, \Gamma$ converges via maps $P^{h}$ to $\mathcal{I}_{\alpha}: X_{\alpha} \rightarrow \mathbb{R}$ iff:

(a) Lower bound: For every $w \in X_{\alpha}$ and every sequence $\left(y^{h}\right)_{h>0} \subset Y$ which $P^{h}$-converges to $w$ as $h \rightarrow 0$ it holds that

$$
\operatorname{linf}_{h \rightarrow 0} \mathcal{I}_{\alpha}^{h}\left(y^{h}\right) \geqslant \mathcal{I}_{\alpha}(w)
$$

(b) Upper bound: For every $w \in X_{\alpha}$ there exists a recovery sequence $\left(y^{h}\right)_{h>0} \subset Y$ which $P^{h}$-converges to $w$ as $h \rightarrow 0$ and

$$
\operatorname{lsup}_{h \rightarrow 0} \mathcal{I}_{\alpha}^{h}\left(y^{h}\right) \leqslant \mathcal{I}_{\alpha}(w)
$$

Finally, we identify what the space of admissible displacements for the limit theories will be:

$$
X_{\alpha}^{0}:= \begin{cases}W_{s h}^{2,2}(\omega ; \mathbb{R}) & \text { if } \quad \alpha \in(2,3) \\ W^{1,2}\left(\omega ; \mathbb{R}^{2}\right) \times W^{2,2}(\omega ; \mathbb{R}) & \text { if } \quad \alpha \geqslant 3\end{cases}
$$

where the space of out-of-plane displacements with singular Hessian

$$
W_{s h}^{2,2}(\omega):=\left\{v \in W^{2,2}(\omega ; \mathbb{R}): \operatorname{det} \nabla^{2} v=0 \text { a.e. }\right\}
$$

\footnotetext{
${ }^{4}$ We refer to the notes [7] for a quick introduction to $\Gamma$-convergence.
} 
will be central in the linearised Kirchhoff theory. We will define the functionals to be $+\infty$ for inadmissible displacements in $X_{\alpha} \backslash X_{\alpha}^{0}$.

\section{MAIN RESULTS}

Our first goal is to prove that in the pre-strained setting described above one has a hierarchy of plate models à la [23]. The proof is split into several theorems in Section 5. For notation we refer to the end of Section 1, for details on our particular use of $\Gamma$-convergence, see Definition 2.3.

Theorem 3.1 (Hierarchy of effective theories). Let

$$
\mathcal{I}_{\alpha}^{h}(y)=\frac{1}{h^{2 \alpha-2}} \int_{\Omega_{1}} W_{\alpha}^{h}\left(x_{3}, \nabla_{h} y(x)\right) \mathrm{d} x .
$$

If $\alpha \in(2,3)$ and $\omega$ is convex, ${ }^{5}$ then the elastic energies $\mathcal{I}_{\alpha}^{h} \Gamma$-converge to the linearised Kirchhoff energy

$$
\mathcal{I}_{\mathrm{IKi}}(v):=\left\{\begin{aligned}
\frac{1}{2} \int_{\omega} \bar{Q}_{2}^{\star}\left(-\nabla^{2} v\right) & \text { if } v \in W_{s h}^{2,2}(\omega), \\
\infty & \text { otherwise }
\end{aligned}\right.
$$

where $\bar{Q}_{2}^{\star}$ is defined in (2.6). See Theorems 5.1 and 5.2.

If $\alpha=3$ and $\theta>0$ then the energies $\mathcal{I}_{\theta}^{h}:=\frac{1}{\theta} \mathcal{I}_{\alpha=3}^{h} \Gamma$-converge to the von Kármán type energy ${ }^{6}$

$$
\mathcal{I}_{\mathrm{vK}}^{\theta}(u, v):=\left\{\begin{array}{c}
\frac{1}{2} \int_{\omega} \bar{Q}_{2}\left(\theta^{1 / 2}\left(\nabla_{s} u+\frac{1}{2} \nabla v \otimes \nabla v\right),-\nabla^{2} v\right) \\
\quad \text { if }(u, v) \in W^{1,2}\left(\omega ; \mathbb{R}^{2}\right) \times W^{2,2}(\omega ; \mathbb{R}), \\
\infty, \text { otherwise, }
\end{array}\right.
$$

where $\bar{Q}_{2}$ is defined in (2.5). See Theorems 5.1 and 5.4 .

Finally, if $\alpha>3$ then $\mathcal{I}_{\alpha}^{h} \Gamma$-converges to the linearised von Kármán energy

$$
\mathcal{I}_{\mathrm{lvK}}(u, v):=\left\{\begin{array}{c}
\frac{1}{2} \int_{\omega} \bar{Q}_{2}\left(\nabla_{s} u,-\nabla^{2} v\right) \\
\quad \text { if }(u, v) \in W^{1,2}\left(\omega ; \mathbb{R}^{2}\right) \times W^{2,2}(\omega ; \mathbb{R}) \\
\infty, \text { otherwise. }
\end{array}\right.
$$

See Theorems 5.1 and 5.5 .

Moreover, in all cases $\alpha>2$ for every sequence $\left(y^{h}\right)_{h>0}$ of finite scaled energy there exists a subsequence (not relabelled) such hat $\left(y^{h}\right)_{h>0} P^{h}$-converges to $v \in X_{\alpha}\left(\right.$ if $\alpha \in(2,3)$ ), respectively $(u, v) \in X_{\alpha}$ (if $\alpha \geq 3$ ), see Lemma 4.1.

\section{Remark 3.2.}

1. We will not be considering body forces for simplicity, but including them in the analysis as in [23] is straightforward.

2. A standard argument shows that almost minimisers of $\mathcal{I}_{\alpha}^{h} P^{h}$-converge (up to subsequences) to minimisers of the limiting functional $\mathcal{I}_{\mathrm{lKi}}$, respectively $\mathcal{I}_{\mathrm{vK}}$, respectively $\mathcal{I}_{\mathrm{lvK}}$.

3. Explicit formulae for $\bar{Q}_{2}^{\star}, \bar{Q}_{2}$ can be found in ([15], Sect. 2.2).

4. Convexity of the domain is required for the representation theorems in Section 7 which are used in the construction of the recovery sequence for $\alpha \in(2,3)$. Thanks to the results in $[25,26]$ the theorem is also true for general simply connected domains whose boundary satisfies a (mild) regularity assumption, more

\footnotetext{
${ }^{5}$ Also certain non-convex domains can be treated, see Remark 3.2.4 below.

${ }^{6}$ Again, we slightly overload the notation in what would be a double definition of $\mathcal{I}_{3}^{h}$, trusting the letter used in the subindex to dispel the ambiguity.
} 
precisely, if there exists $\Sigma=\bar{\Sigma} \subset \partial \omega$ with $\mathcal{H}^{1}(\Sigma)=0$ such that on its complement $\partial \omega \backslash \Sigma$ the outer unit normal to $\omega$ exists and is continuous. This is verified, e.g., if $\partial \omega$ is piecewise continuously differentiable. For the sake of clarity we explicitly address the case of convex $\omega$, but we do include all the necessary arguments to cover the other case as well.

The functional $\mathcal{I}_{1 \mathrm{Ki}}$ is said to model a linearised Kirchhoff regime because the isometry condition $\nabla^{\top} y \nabla y=I$ of the Kirchhoff model is replaced by $\operatorname{det} \nabla^{2} v=0$, a necessary and sufficient condition for the existence of an in-plane displacement $u$ such that $\nabla u+\nabla^{\top} u+\nabla v \otimes \nabla v=0$. This condition is to leading order equivalent to $\nabla^{\top} y \nabla y=I$ for deformations $y=\left(h^{2 \alpha-4} u, h^{\alpha-2} v\right) .{ }^{7}$ The functional $\mathcal{I}_{\mathrm{vK}}^{\theta}$ is of von Kármán type with in-plane and out-of-plane strains interacting in a membrane energy term, and a bending energy term. For simple choices of $Q_{2}$ and $B^{h}$, one recovers the classical functional (1.1). Finally, we say that the third limit $\mathcal{I}_{\mathrm{lvK}}$, models a linearised von Kármán (or fully linear) regime by analogy with the classical equivalent, but it is of a different kind than the one expected from the hierarchy derived in [23], since it again features an interplay between in-plane and out-of-plane components. ${ }^{8}$

Our second goal is to show that the limit energy $\mathcal{I}_{\mathrm{vK}}^{\theta}$ interpolates between $\mathcal{I}_{\mathrm{lKi}}$ and $\mathcal{I}_{\mathrm{lvK}}$ as the parameter $\theta$ moves from $\infty$ to 0 , so that one can say that the theory of von Kármán type bridges the other two. More precisely, in Section 6 we prove:

Theorem 3.3 (Interpolating regime). The following two $\Gamma$-limits hold:

$$
\mathcal{I}_{\mathrm{vK}}^{\theta} \underset{\theta \uparrow \infty}{\stackrel{\Gamma}{\longrightarrow}} \mathcal{I}_{\mathrm{lKi}}
$$

if $\omega$ is convex ${ }^{9}$ (Thms. 6.4 and 6.5) and:

$$
\mathcal{I}_{\mathrm{vK}}^{\theta} \underset{\theta \downarrow 0}{\stackrel{\Gamma}{\longrightarrow}} \mathcal{I}_{\mathrm{lvK}}
$$

(Thm. 6.6 and 6.7). Furthermore, sequences $\left(u_{\theta}, v_{\theta}\right)_{\theta>0}$ of bounded energy $\mathcal{I}_{\mathrm{vK}}^{\theta}$ are precompact in suitable spaces as $\theta \uparrow \infty$ or $\theta \downarrow 0$ (Thm. 6.3).

Example. The easiest non-trivial situation is given by a linear internal misfit in a homogeneous material with

$$
B(t):=t I_{3} \in \mathbb{R}^{3 \times 3} \text { and } Q_{2}(t, \cdot)=Q_{2}(\cdot)
$$

Then

$$
\mathcal{I}_{\mathrm{vK}}^{\theta}(u, v)=\frac{\theta}{2} \int_{\omega} Q_{2}\left(\nabla_{s} u+\frac{1}{2} \nabla v \otimes \nabla v\right)+\frac{1}{24} \int_{\omega} Q_{2}\left(\nabla^{2} v-I\right) .
$$

for $(u, v) \in W^{1,2}\left(\omega ; \mathbb{R}^{2}\right) \times W^{2,2}(\omega ; \mathbb{R})$. We refer to $[14,15]$ for more worked out examples.

\section{Compactness And identification of Limit STRAin}

We collect here some basic results proving compactness of sequences of finite scaled energy and providing explicit representations for the limit strains, as required for the proofs of $\Gamma$-convergence in Section 3 . These

\footnotetext{
${ }^{7}$ In the numerical analysis literature, the denomination linear Kirchhoff is sometimes used for a pure bending regime without constraints.

${ }^{8}$ This is in contrast to [23]. In our setting with the additional dependence on the $x_{3}$ coordinate, it is not possible to simply drop terms while bounding below the energy in the proof of the lower bound as is done in ([23], p. 211). Indeed, our keeping track of both in-plane and out-of-plane displacements is essential to capture the effect of pre-stressing with the internal misfit $B^{h}$.

${ }^{9}$ Or simply connected with $\partial \omega$ satisfying the condition in Remark 3.2.4
} 
results are direct consequences of the homogeneous case treated in ([23], Lem. 1). We recall the definition of the scaled elastic energies (2.1):

$$
\mathcal{I}_{\alpha}^{h}(y)=\frac{1}{h^{2 \alpha-2}} \int_{\Omega_{1}} W_{0}\left(x_{3}, \nabla_{h} y(x)\left(I+h^{\alpha-1} B^{h}\left(x_{3}\right)\right)\right) \mathrm{d} x .
$$

Lemma 4.1. Let $\alpha \in(2, \infty)$ and let $\left(y^{h}\right)_{h>0} \subset Y$ have finite scaled energy $\mathcal{I}_{\alpha}^{h}$. For every $h>0$ there exist constants $\bar{R}^{h} \in \mathrm{SO}(3)$ and $c^{h} \in \mathbb{R}^{3}$ such for the corrected deformations

$$
\tilde{y}^{h}=\rho\left(y^{h}\right):=\left(\bar{R}^{h}\right)^{\top} y^{h}-c^{h}
$$

there exist rotations $R^{h}: \omega \rightarrow \mathrm{SO}(3)$ (extended constantly along $x_{3}$ to all of $\Omega_{1}$ outside $\left.\{0\} \times \omega\right)$ approximating $\nabla_{h} \tilde{y}^{h}$ in $L^{2}\left(\Omega_{1}\right)$. Quantitatively:

$$
\left\|\nabla_{h} \tilde{y}^{h}-R^{h}\right\|_{0,2, \Omega_{1}} \leqslant C h^{\alpha-1}
$$

Furthermore,

$$
\left\|R^{h}-I\right\|_{0,2, \Omega_{1}} \leqslant C h^{\alpha-2}
$$

Finally there exists a subsequence (not relabelled) such that for the scaled and averaged in-plane and out-of-plane displacements from (2.8) there exist $(u, v) \in W^{1,2}\left(\omega ; \mathbb{R}^{2}\right) \times W^{2,2}(\omega)$ such that, if $\alpha \neq 3$ :

$$
u_{\alpha}^{h} \rightarrow u \text { in } W^{1,2}\left(\omega ; \mathbb{R}^{2}\right) \quad \text { and } \quad v_{\alpha}^{h} \rightarrow v \text { in } W^{1,2}(\omega),
$$

If $\alpha=3$ an analogous result holds with $u_{\theta}^{h}$ and $v_{\theta}^{h}$ from (2.9).

In particular, in the sense of Definition 2.2 we have that $\left(y^{h}\right)_{h>0} P^{h}$-converges to $v \in X_{\alpha}$ (if $\alpha \in(2,3)$ ), respectively $(u, v) \in X_{\alpha}$ (if $\alpha \geq 3$ ).

Proof. This is exactly a particular case of ([23], Lem. 1), estimates (84) and (85) and estimates (86) and (87), once we prove that if $\left(y^{h}\right)_{h>0}$ have finite scaled $\mathcal{I}_{\alpha}^{h}$ energy, then they have finite scaled energy in the sense of [23].

Note first that among all choices we can make for the energy density $W$ which fulfil the assumptions in [23], we can pick $\operatorname{dist}^{2}(\cdot, \mathrm{SO}(3))$. Therefore we will bound this quantity. Write $\mathrm{d}(F):=\operatorname{dist}(F, \mathrm{SO}(3))$. We begin by using Assumption 2.1.e:

$$
\begin{aligned}
C h^{2 \alpha-2} & \geqslant \int_{\Omega_{1}} W_{0}\left(x_{3}, \nabla_{h} y(x)\left(I+h^{\alpha-1} B^{h}\left(x_{3}\right)\right)\right) \\
& \gtrsim \int_{\Omega_{1}} \mathrm{~d}^{2}\left(\nabla_{h} y(x)\left(I+h^{\alpha-1} B^{h}\left(x_{3}\right)\right)\right) .
\end{aligned}
$$

Consider now the following:

$$
\begin{aligned}
\mathrm{d}^{2}\left(F\left(I+h^{\alpha-1} B^{h}\right)\right) & \geqslant \frac{1}{2} \mathrm{~d}^{2}(F)-\left|F h^{\alpha-1} B^{h}\right|^{2} \\
& \geqslant \frac{1}{2} \mathrm{~d}^{2}(F)-C h^{2 \alpha-2}\left|1+\mathrm{d}^{2}(F)\right| \\
& \geqslant \frac{1}{4} \mathrm{~d}^{2}(F)-C h^{2 \alpha-2} .
\end{aligned}
$$


But then we are done since:

$$
h^{2 \alpha-2} \gtrsim \int_{\Omega_{1}} \frac{1}{4} \mathrm{~d}^{2}\left(\nabla_{h} y\right)
$$

Lemma 4.2. ${ }^{10}$ Let $\alpha \in(2, \infty)$ and let $\left(y^{h}\right)_{h>0}$ be a sequence in $Y$ which $P^{h}$-converges to $(u, v) \in X_{\alpha}$ in the sense of Theorem 3.1 and $R^{h}: \omega \rightarrow \mathrm{SO}(3)$ (extended constantly along $x_{3}$ to all of $\Omega_{1}$ outside $\{0\} \times \omega$ ) such that

$$
\left\|\nabla_{h} y^{h}-R^{h}\right\|_{0,2, \Omega_{1}} \leqslant C h^{\alpha-1}
$$

Then:

$$
A^{h}:=\frac{1}{h^{\alpha-2}}\left(R^{h}-I\right) \longrightarrow \begin{cases}\sqrt{\theta} A & \text { if } \quad \alpha=3, \\ A & \text { else, }\end{cases}
$$

where

$$
A=e_{3} \otimes \hat{\nabla} v-\hat{\nabla} v \otimes e_{3}
$$

and

$$
G^{h}:=\frac{\left(R^{h}\right)^{\top} \nabla_{h} y^{h}-I}{h^{\alpha-1}} \rightarrow G \text { in } L^{2}\left(\Omega_{1} ; \mathbb{R}^{3 \times 3}\right)
$$

where the submatrix $\check{G} \in \mathbb{R}^{2 \times 2}$ is affine in $x_{3}$ :

$$
\check{G}\left(x^{\prime}, x_{3}\right)=G_{0}\left(x^{\prime}\right)+x_{3} G_{1}\left(x^{\prime}\right)
$$

and

$$
\begin{gathered}
G_{1}=\left\{\begin{aligned}
-\sqrt{\theta} \nabla^{2} v & \text { if } \alpha=3, \\
-\nabla^{2} v & \text { else, }
\end{aligned}\right. \\
\operatorname{sym} G_{0}=\left\{\begin{aligned}
\theta\left(\nabla_{s} u+\frac{1}{2} \nabla v \otimes \nabla v\right) & \text { if } \alpha=3, \\
\nabla_{s} u & \text { if } \alpha>3,
\end{aligned}\right.
\end{gathered}
$$

and

$$
\nabla_{s} u+\frac{1}{2} \nabla v \otimes \nabla v=0, \text { if } \alpha \in(2,3)
$$

Proof. See ([23], pp. 208-209).

\footnotetext{
${ }^{10}$ This is almost word for word ([23], Lem. 2) with the very minor addition of the factors $\theta, \sqrt{\theta}$. For other scaling choices see ([23], p. 208). Note that this is inspired by ([9], Thm. 5.4.2) (itself based in ([9], Thm. 1.4.1.c)).
} 


\section{5. $\Gamma$-CONVERGENCE OF THE HIERARCHY}

This section proves the lower (Thm. 5.1) and upper bounds (Thm. 5.2, 5.4 and 5.5) required for deriving the hierarchy of models in Theorem 3.1. Recall that we are always using weak convergence in the spaces $X_{\alpha}$.

Theorem 5.1 (Lower bounds). Let $\alpha \in(2,3)$. If $\left(y^{h}\right)_{h>0} \subset Y$ is a sequence $P_{\alpha}^{h}$-converging to $v \in X_{\alpha}$, then

$$
\operatorname{linf}_{h \rightarrow 0} \mathcal{I}_{\alpha}^{h}\left(y^{h}\right) \geqslant \mathcal{I}_{\mathrm{lKi}}(v) .
$$

Now let $\alpha=3$. If $\left(y^{h}\right)_{h>0} \subset Y$ is a sequence $P_{\theta}^{h}$-converging to $(u, v) \in X_{\alpha}$, then for all $\theta>0$

$$
\operatorname{linf}_{h \rightarrow 0} \frac{1}{\theta} \mathcal{I}_{\alpha}^{h}\left(y^{h}\right) \geqslant \mathcal{I}_{\mathrm{vK}}^{\theta}(u, v) .
$$

Finally, let $\alpha>3$. If $\left(y^{h}\right)_{h>0} \subset Y$ is a sequence $P_{\alpha}^{h}$-converging to $(u, v) \in X_{\alpha}$, then

$$
\operatorname{linf}_{h \rightarrow 0} \mathcal{I}_{\alpha}^{h}\left(y^{h}\right) \geqslant \mathcal{I}_{\mathrm{lvK}}(u, v) .
$$

Proof. If $\alpha=3$, we define $\tilde{B}^{h}:=\sqrt{\theta} B^{h}$ and $\tilde{B}=\sqrt{\theta} B$, otherwise $\tilde{B}:=B$ and $\tilde{B}^{h}:=B^{h}$. Following closely the techniques in $[22,23,51,52]$ we use a Taylor expansion of the energy around the identity which allows us to cancel or identify its lower order terms. For this we must correct the deformations with an approximation by rotations and work in adequate sets where there is control over higher order terms.

Upon passing to a subsequence (not relabelled) which realises $\operatorname{linf}_{h \rightarrow 0} \mathcal{I}_{\alpha}^{h}\left(y^{h}\right)$ as its limit, we may w.l.o.g. assume that $\left(y^{h}\right)_{h>0}$ has finite scaled $\mathcal{I}_{\alpha}^{h}$ energy and pass to further subsequences in the following.

Step 1: Approximation by rotations. We will be working with the corrected deformations

$$
\rho\left(y^{h}\right):=\left(\bar{R}^{h}\right)^{\top} y^{h}-c^{h}
$$

as given in Lemma 4.1. For simplicity we use the same notation $y^{h}$ for these functions. Also by Lemma 4.1 there exist rotations $R^{h}: \omega \rightarrow \mathrm{SO}(3)$ (extended constantly along $x_{3}$ to all of $\Omega_{1}$ outside $\omega \times\{0\}$ ) which approximate $\nabla_{h} y^{h}$ in $L^{2}\left(\Omega_{1}\right)$ and are close to the identity, as required for the identification of the limit strain in Lemma 4.2. Step 2: Rewriting of the deformation gradient. The functions

$$
G^{h}:=\frac{\left(R^{h}\right)^{\top} \nabla_{h} y^{h}-I}{h^{\alpha-1}}
$$

are uniformly bounded in $L^{2}$ by invariance of the norm by rotations:

$$
\left\|G^{h}\right\|_{0,2, \Omega_{1}}=h^{1-\alpha}\left\|\nabla_{h} y^{h}-R^{h}\right\|_{0,2, \Omega_{1}} \leqslant C .
$$

Now, by the frame invariance of $W^{h}\left(x_{3}, \cdot\right)$

$$
\begin{aligned}
W^{h}\left(x_{3}, \nabla_{h} y^{h}\right) & =W^{h}\left(x_{3},\left(R^{h}\right)^{\top} \nabla_{h} y^{h}\right) \\
& =W_{0}\left(x_{3},\left(R^{h}\right)^{\top} \nabla_{h} y^{h}\left(I+h^{\alpha-1} \tilde{B}^{h}\left(x_{3}\right)\right)\right) \\
& =W_{0}\left(x_{3}, I+h^{\alpha-1} A^{h}\right),
\end{aligned}
$$

where we have set

$$
\begin{aligned}
A^{h}(x) & :=\frac{\left(R^{h}\right)^{\top} \nabla_{h} y^{h}(x)-I}{h^{\alpha-1}}+\left(R^{h}\right)^{\top} \nabla_{h} y^{h}(x) \tilde{B}^{h}\left(x_{3}\right) \\
& =G^{h}+\left(R^{h}\right)^{\top} \nabla_{h} y^{h} \tilde{B}^{h} .
\end{aligned}
$$


Step 3: Cutoff function. Let $\chi^{h}$ be the characteristic function of the "good set" $\left\{x \in \Omega_{1}:\left|G^{h}\right| \leqslant h^{-1 / 2}\right\}$, where $\overline{\left(R^{h}\right)^{\top} \nabla_{h} y^{h} \text { is close to } I}$. Here we have:

$$
h^{1 / 2} \gg h^{\alpha-3 / 2} \geqslant \chi^{h}\left|h^{\alpha-1} G^{h}\right|=\chi^{h}\left|\left(R^{h}\right)^{\top} \nabla_{h} y^{h}-I\right|=\chi^{h}\left|\nabla_{h} y^{h}-R^{h}\right|,
$$

which, because $\left|R^{h}\right| \equiv \sqrt{3}$, implies that $\chi^{h}\left|\nabla_{h} y^{h}\right| \leqslant C$. Consequently, since the $\tilde{B}^{h}$ are uniformly bounded as well:

$$
\begin{aligned}
\chi^{h}\left|h^{\alpha-1} A^{h}\right| & =\chi^{h}\left|h^{\alpha-1} G^{h}+h^{\alpha-1}\left(R^{h}\right)^{\top} \nabla_{h} y^{h} \tilde{B}^{h}\right| \\
& \leqslant \chi^{h}\left|h^{\alpha-1} G^{h}\right|+\mathcal{O}\left(h^{\alpha-1}\right) \\
& =o\left(h^{1 / 2}\right),
\end{aligned}
$$

and then

$$
\operatorname{dist}\left(I+h^{\alpha-1} \chi^{h} A^{h}, \mathrm{SO}(3)\right) \leqslant\left|I+h^{\alpha-1} \chi^{h} A^{h}-I\right|=o\left(h^{1 / 2}\right),
$$

so in the good sets we may indeed expand around $I$ for small values of $h$. Now, the sequence $\left(G^{h}\right)_{h>0}$ is bounded in $L^{2}$ by (5.1) so we may extract a subsequence converging weakly in $L^{2}$ to some $G \in L^{2}\left(\Omega_{1}\right)$, which we consider from now on without relabelling. Furthermore the sequence $\left(\chi^{h}\right)_{h>0}$ is essentially bounded and $\chi^{h} \rightarrow 1$ in measure in $\Omega_{1}$. Indeed $\left|\left\{\left|\chi^{h}-1\right|>\varepsilon\right\}\right|=\left|\left\{\left|G^{h}\right|>h^{-1 / 2}\right\}\right| \rightarrow 0$ as $h \rightarrow 0$ because $\left\|G^{h}\right\|_{0,2, \Omega_{1}} \leqslant C$ uniformly. Consequently we have

$$
\chi^{h} G^{h} \rightarrow G \text { in } L^{2}\left(\Omega_{1}\right)
$$

Analogously, the sequence $\left(\chi^{h} \tilde{B}^{h}\right)_{h>0}$ is essentially bounded and converges in measure to $\tilde{B}$ because $\mid\left\{\mid \chi^{h} \tilde{B}^{h}-\right.$ $\tilde{B} \mid>\varepsilon\}|\leqslant|\left\{\left|\tilde{B}^{h}-\tilde{B}\right|>\varepsilon\right\}|+|\left\{\chi^{h}=0\right\} \cap\{|\tilde{B}|>\varepsilon\} \mid \rightarrow 0$. Hence, using again the strong convergence $\left(R^{h}\right)^{\top} \nabla_{h} y^{h} \rightarrow I$ in $L^{2}\left(\Omega_{1}\right)$ (Lem. 4.1$)$ :

$$
\left(R^{h}\right)^{\top} \nabla_{h} y^{h} \chi^{h} \tilde{B}^{h} \rightarrow \tilde{B} \text { in } L^{2}\left(\Omega_{1}\right) .
$$

So we conclude

$$
\chi^{h} A^{h} \rightarrow A:=G+\tilde{B} \text { in } L^{2}\left(\Omega_{1}\right) .
$$

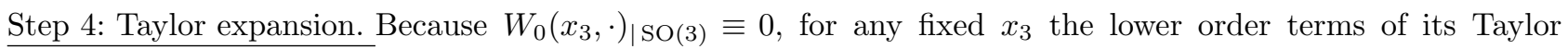
expansion

$$
W_{0}\left(x_{3}, I+E\right)=W_{0}\left(x_{3}, I\right)+D W_{0}\left(x_{3}, I\right)[E]+\frac{1}{2} D^{2} W_{0}\left(x_{3}, I\right)[E, E]+o\left(|E|^{2}\right)
$$

vanish and we have (for small enough $h$, as explained above)

$$
W_{0}\left(x_{3}, I+h^{\alpha-1} \chi^{h} A^{h}\right)=\frac{1}{2} Q_{3}\left(x_{3}, h^{\alpha-1} \chi^{h} A^{h}\right)+\eta^{h}\left(x_{3}, h^{\alpha-1} \chi^{h} A^{h}\right),
$$

where $\eta^{h}\left(x_{3}, h^{\alpha-1} \chi^{h} A^{h}\right)=o\left(h^{2 \alpha-2}\left|\chi^{h} A^{h}\right|^{2}\right)$ represents the higher order terms. Defining the uniform bound

$$
\omega(s):=\operatorname{esssup}_{-1 \leqslant 2 r \leqslant 1|M| \leqslant s} \sup \left|\eta^{h}(r, M)\right|,
$$


we have $\omega(s)=o\left(s^{2}\right)$ by Assumption 2.1.c, and integrating over the rescaled domain $\Omega_{1}$ we obtain the estimate:

$$
\begin{aligned}
& \frac{1}{h^{2 \alpha-2}} \int_{\Omega_{1}} W^{h}\left(x_{3}, \nabla_{h} y^{h}\right) \mathrm{d} x \\
& \geqslant \frac{1}{h^{2 \alpha-2}} \int_{\Omega_{1}} W^{h}\left(x_{3}, I+\chi^{h} h^{\alpha-1} A^{h}\right) \mathrm{d} x \\
& \geqslant \frac{1}{h^{2 \alpha-2}} \int_{\Omega_{1}} \frac{h^{2 \alpha-2}}{2} Q_{3}\left(x_{3}, \chi^{h} A^{h}\right)-\omega\left(\left|h^{\alpha-1} \chi^{h} A^{h}\right|\right) \mathrm{d} x \\
& \quad=\frac{1}{2} \int_{\Omega_{1}} Q_{3}\left(x_{3}, \chi^{h} A^{h}\right)-\frac{1}{h^{2 \alpha-2}} \int_{\Omega_{1}} \omega\left(\left|h^{\alpha-1} \chi^{h} A^{h}\right|\right) \mathrm{d} x .
\end{aligned}
$$

Step 5: The limit inferior. In order to pass to the limit, for the first integral on the right hand side of (5.2) we use that $Q_{3}$ is positive semidefinite, therefore convex and continuous, and the integral is sequentially weakly lower semicontinuous. For the second integral we use again Assumption 2.1.c and the fact that $\left|h^{\alpha-1} \chi^{h} A^{h}\right| \leqslant h^{1 / 2}$ to obtain the bound (uniform over $\Omega_{1}$ ):

$$
\frac{\omega\left(\left|h^{\alpha-1} \chi^{h} A^{h}\right|\right)}{\left|h^{\alpha-1} \chi^{h} A^{h}\right|^{2}} \leqslant \sup _{|s| \leqslant h^{1 / 2}} \frac{\omega(s)}{s^{2}} \longrightarrow 0 \text { as } h \rightarrow 0 .
$$

But then, because $\chi^{h} A^{h}$ converges weakly in $L^{2}$, we have $\left\|\chi^{h} A^{h}\right\|_{0,2, \Omega_{1}}^{2} \leqslant C$ and

$$
\begin{aligned}
\frac{1}{h^{2 \alpha-2}} \int_{\Omega_{1}} \omega\left(\left|h^{\alpha-1} \chi^{h} A^{h}\right|\right) \mathrm{d} x & =\int_{\Omega_{1}} \frac{\omega\left(\left|h^{\alpha-1} \chi^{h} A^{h}\right|\right)}{\left|h^{\alpha-1} \chi^{h} A^{h}\right|^{2}} \frac{\left|h^{\alpha-1} \chi^{h} A^{h}\right|^{2}}{h^{2 \alpha-2}} \mathrm{~d} x \\
& \leqslant \sup _{|s| \leqslant h^{1 / 2}} \frac{\omega(s)}{s^{2}} \underbrace{\int_{\Omega_{1}}\left|\chi^{h} A^{h}\right|^{2} \mathrm{~d} x}_{\text {uniformly bded. }} \longrightarrow 0
\end{aligned}
$$

as $h \rightarrow 0$. Taking the lim inf at both sides of (5.2) we have:

$$
\begin{aligned}
\operatorname{linf}_{h \rightarrow 0} \frac{1}{h^{2 \alpha-2}} \int_{\Omega_{1}} W^{h}\left(x_{3}, \nabla_{h} y^{h}\right) \mathrm{d} x \geqslant & \operatorname{linf}_{h \rightarrow 0} \frac{1}{2} \int_{\Omega_{1}} Q_{3}\left(x_{3}, \chi^{h} A^{h}\right) \mathrm{d} x \\
& -\lim _{h \rightarrow 0} \frac{1}{h^{2 \alpha-2}} \int_{\Omega_{1}} \omega\left(\left|h^{\alpha-1} A^{h}\right|\right) \mathrm{d} x \\
\geqslant & \frac{1}{2} \int_{\Omega_{1}} Q_{3}\left(x_{3}, G+\tilde{B}\right) \mathrm{d} x \\
\geqslant & \frac{1}{2} \int_{\Omega_{1}} Q_{2}\left(x_{3}, \check{G}+\check{\tilde{B}}\right) \mathrm{d} x
\end{aligned}
$$

where the last estimate follows trivially from the definition of $Q_{2}$.

By Lemma 4.2 the limit strain $\check{G}$ has the representation

$$
\check{G}(x)=G_{0}\left(x^{\prime}\right)+x_{3} G_{1}\left(x^{\prime}\right),
$$


with $G_{1}$ and sym $G_{0}$ as in Lemma 4.2 . We plug both into the last integral and use the fact that $Q_{2}\left(x_{3}, \cdot\right)$ vanishes on antisymmetric matrices to obtain

$$
\begin{aligned}
\operatorname{linf}_{h \rightarrow 0} & \frac{1}{h^{2 \alpha-2}} \int_{\Omega_{1}} W_{\alpha}^{h}\left(x_{3}, \nabla_{h} y^{h}\right) \mathrm{d} x \\
& \geqslant \frac{1}{2} \int_{\Omega_{1}} Q_{2}\left(x_{3}, G_{0}\left(x^{\prime}\right)+x_{3} G_{1}\left(x^{\prime}\right)+\check{\tilde{B}}\left(x_{3}\right)\right) \mathrm{d} x \\
& =\frac{1}{2} \int_{\omega} \bar{Q}_{2}\left(\operatorname{sym} G_{0}, G_{1}\right) \mathrm{d} x^{\prime}
\end{aligned}
$$

with with $G_{1}$ and sym $G_{0}$ given respectively by (4.2) and (4.3). In particular, if $\alpha=3$, we have again:

$$
\begin{aligned}
\operatorname{limf}_{h \rightarrow 0} \frac{1}{\theta h^{4}} \int_{\Omega_{1}} W_{\alpha}^{h}\left(x_{3}, \nabla_{h} y^{h}\right) \mathrm{d} x & \geqslant \frac{1}{2 \theta} \int_{\omega} \bar{Q}_{2}\left(\operatorname{sym} G_{0}, G_{1}\right) \mathrm{d} x^{\prime} \\
& =\frac{1}{2} \int_{\omega} \bar{Q}_{2}\left(\theta^{1 / 2}\left(\nabla_{s} u+\frac{1}{2} \nabla v \otimes \nabla v\right),-\nabla^{2} v\right) .
\end{aligned}
$$

If $\alpha \in(2,3)$, then sym $G_{0}$ is unknown, so we must further relax the integrand. With the definition of $\bar{Q}_{2}^{\star}$ we see that the final integral above is

$$
\frac{1}{2} \int_{\Omega_{1}} Q_{2}\left(x_{3}, G_{0}-x_{3} \nabla^{2} v+\check{B}\right) \mathrm{d} x \geqslant \frac{1}{2} \int_{\omega} \bar{Q}_{2}^{\star}\left(-\nabla^{2} v\right) \mathrm{d} x^{\prime}
$$

We proceed now with the computation of the recovery sequences for each of the three regimes discussed.

Theorem 5.2 (Upper bound, linearised Kirchhoff regime). Assume $\omega$ is convex ${ }^{11}$, let $\alpha \in(2,3)$ and $v \in X_{\alpha}:=$ $W^{1,2}(\omega)$. There exists a sequence $\left(y^{h}\right)_{h>0} \subset Y$ which $P^{h}$-converges to $v$ such that

$$
\operatorname{lsup}_{h \rightarrow 0} \mathcal{I}_{\alpha}^{h}\left(y^{h}\right) \leqslant \mathcal{I}_{1 \mathrm{Ki}}(v)
$$

with $\mathcal{I}_{\mathrm{lKi}}$ defined as in $(3.1)$ by

$$
\mathcal{I}_{\mathrm{lKi}}(v):=\left\{\begin{aligned}
\frac{1}{2} \int_{\omega} \bar{Q}_{2}^{\star}\left(\nabla^{2} v\left(x^{\prime}\right)\right) \mathrm{d} x^{\prime} & \text { if } v \in W_{s h}^{2,2}(\omega), \\
\infty & \text { otherwise. }
\end{aligned}\right.
$$

Proof. We set $\varepsilon=h^{\alpha-2}$, so that $h \ll \varepsilon \ll 1$ and $h^{2} \ll \varepsilon h \ll 1$.

Step 1: Setup and recovery sequence. The functional $\mathcal{I}_{1 \mathrm{Ki}}$ is strongly continuous on $W_{s h}^{2,2}(\omega)$ by the continuity and 2-growth of $\bar{Q}_{2}^{\star}$. By Theorem 7.1 we have a set $\mathcal{V}_{0}$ of smooth maps with singular Hessian which is $W^{2,2_{-}}$ dense in $W_{s h}^{2,2}$, see (7.2). Therefore, by a standard argument (see, e.g., [7]) it is enough to construct here the recovery sequence. Take then a smooth function $v \in \mathcal{V}_{0}$. Because $\|\nabla v\|_{\infty}<C$, for $\varepsilon$ small enough there exist by ([23], Thm. 7) in-plane displacements $u_{\varepsilon} \in W^{2,2}\left(\omega ; \mathbb{R}^{2}\right) \cap W^{2, \infty}\left(\omega ; \mathbb{R}^{2}\right)$ with uniform bounds in $\varepsilon$ such that the deformations

$$
\bar{y}_{\varepsilon}\left(x^{\prime}\right):=\left(\begin{array}{c}
x^{\prime}+\varepsilon^{2} u_{\varepsilon}\left(x^{\prime}\right) \\
\varepsilon v\left(x^{\prime}\right)
\end{array}\right)
$$

\footnotetext{
${ }^{11}$ Instead of convexity one may assume that $\omega$ be simply connected and $\partial \omega$ satisfy the assumption detailed in Remark 3.2.4, because Theorem 7.1, Corollary 7.4 and ([23], Thm. 7) also apply in this situation.
} 
are isometries. ${ }^{12}$ That is: $\nabla^{\top} \bar{y}_{\varepsilon} \nabla \bar{y}_{\varepsilon}=I_{2}$, where

$$
\nabla \bar{y}_{\varepsilon}=\left(\begin{array}{cc}
I_{2} \\
0 & 0
\end{array}\right)+\varepsilon\left(\begin{array}{c}
0_{2} \\
\nabla^{\top} v
\end{array}\right)+\varepsilon^{2}\left(\begin{array}{cc}
\nabla u_{\varepsilon} \\
0 & 0
\end{array}\right) \in \mathbb{R}^{3 \times 2} .
$$

Additionally the following normal vectors are unitary in $\mathbb{R}^{3}$ :

$$
\begin{aligned}
b_{\varepsilon}\left(x^{\prime}\right) & :=\bar{y}_{\varepsilon, 1}\left(x^{\prime}\right) \wedge \bar{y}_{\varepsilon, 2}\left(x^{\prime}\right) \\
& =-\varepsilon\left(\begin{array}{c}
\nabla v \\
0
\end{array}\right)+\left(\begin{array}{c}
\varepsilon^{3} \nabla u_{\varepsilon 2} \cdot\left(v_{, 2},-v_{, 1}\right) \\
\varepsilon^{3} \nabla u_{\varepsilon 1} \cdot\left(-v_{, 2}, v_{, 1}\right) \\
1+\varepsilon^{2} \operatorname{tr} \nabla u_{\varepsilon}+\varepsilon^{4} \operatorname{det} \nabla u_{\varepsilon}
\end{array}\right) \\
& =e_{3}-\varepsilon \hat{\nabla} v\left(x^{\prime}\right)+r_{\varepsilon}\left(x^{\prime}\right)
\end{aligned}
$$

where the rest $r_{\varepsilon}$ satisfies

$$
\left\|r_{\varepsilon}\right\|_{1, \infty}=\mathcal{O}\left(\varepsilon^{2}\right)
$$

by virtue of $\left\|u_{\varepsilon}\right\|_{2, \infty} \leqslant C$ and $\|\nabla v\|_{\infty} \leqslant C$. Consequently the matrices

$$
R_{\varepsilon}:=\left(\nabla \bar{y}_{\varepsilon}, b_{\varepsilon}\right)=I+\varepsilon\left(\begin{array}{cc}
0 & -\nabla v \\
\nabla^{\top} v & 0
\end{array}\right)+\underbrace{r_{\varepsilon} \otimes e_{3}+\varepsilon^{2} \hat{\nabla} u_{\varepsilon}}_{=: \tilde{r}_{\varepsilon}}
$$

are in $\mathrm{SO}(3)$ for every $x^{\prime} \in \omega$, with the remaining matrix $\tilde{r}_{\varepsilon}$ satisfying

$$
\left\|\tilde{r}_{\varepsilon}\right\|_{1, \infty}=\mathcal{O}\left(\varepsilon^{2}\right)
$$

by the same arguments as before. Now, for some smooth functions $\alpha, g_{1}, g_{2} \in C^{\infty}(\bar{\omega} ; \mathbb{R}), g:=\left(g_{1}, g_{2}\right)$ and $d \in L^{\infty}\left(\Omega_{1} ; \mathbb{R}^{3}\right)$ with $\nabla^{\prime} d \in L^{\infty}\left(\Omega_{1} ; \mathbb{R}^{3 \times 2}\right)$ and $D \in C^{\infty}\left(\bar{\Omega}_{1} ; \mathbb{R}^{3}\right)$ to be determined later, set

$$
\begin{aligned}
y^{h}\left(x^{\prime}, x_{3}\right):= & \bar{y}_{\varepsilon}\left(x^{\prime}\right)+h\left(x_{3}-\alpha\left(x^{\prime}\right)\right) b_{\varepsilon}\left(x^{\prime}\right)+\varepsilon h\left(g\left(x^{\prime}\right), 0\right) \\
& +\varepsilon h^{2} \int_{0}^{x_{3}} d\left(x^{\prime}, \xi\right) \mathrm{d} \xi+h^{2} D\left(x^{\prime}, x_{3}\right) .
\end{aligned}
$$

We will prove

$$
\mathcal{I}_{\alpha}^{h}\left(y^{h}\right) \underset{h \rightarrow 0}{\longrightarrow} \mathcal{I}_{1 \mathrm{Ki}}(v)
$$

as well as $P_{\alpha}^{h}\left(y^{h}\right) \rightarrow v$ in $W^{1,2}$ for $R^{h} \equiv I \in \mathrm{SO}(3), c^{h} \equiv 0 \in \mathbb{R}^{3}$.

Step 2: Preliminary computations. In order to compute the limit of $\frac{1}{h^{2 \alpha-2}} \int_{\Omega_{1}} W_{0}\left(x_{3}, \nabla_{h} y^{h}\left(I+\varepsilon h B^{h}\right)\right)$ we start with the gradient of the recovery sequence:

$$
\begin{aligned}
\nabla_{h} y^{h}= & \left(\nabla \bar{y}_{\varepsilon}, 0\right)+h \nabla_{h}\left[\left(x_{3}-\alpha\right) b_{\varepsilon}\right] \\
& +\varepsilon h\left[\hat{\nabla} g+d \otimes e_{3}\right]+h D_{, 3} \otimes e_{3}+o(\varepsilon h) .
\end{aligned}
$$

\footnotetext{
${ }^{12}$ The uniform bounds for $\left\|u_{\varepsilon}\right\|_{2,2}$ follow from ([23], Thm. 7), equation (181), and those for $\left\|u_{\varepsilon}\right\|_{2, \infty}$ from the explicit construction done in the proof, in particular equations (183), (186) and (190).
} 
For the first term in $h$ we have

$$
\begin{aligned}
\nabla_{h}\left[\left(x_{3}-\alpha\right) b_{\varepsilon}\right] & =\left(x_{3}-\alpha\right) \hat{\nabla} b_{\varepsilon}-b_{\varepsilon} \otimes \hat{\nabla} \alpha+\frac{1}{h} b_{\varepsilon} \otimes e_{3} \\
& =\left(\alpha-x_{3}\right)\left(\varepsilon \hat{\nabla}^{2} v-\hat{\nabla} r_{\varepsilon}\right)-b_{\varepsilon} \otimes \hat{\nabla} \alpha+\frac{1}{h} b_{\varepsilon} \otimes e_{3}
\end{aligned}
$$

Substituting back into the gradient yields:

$$
\begin{aligned}
\nabla_{h} y^{h}= & R_{\varepsilon}+\varepsilon h \underbrace{\left[\left(\alpha-x_{3}\right) \hat{\nabla}^{2} v+\hat{\nabla} g+d \otimes e_{3}+o(1)\right]}_{=: A^{h}} \\
& -h b_{\varepsilon} \otimes \hat{\nabla} \alpha+h D_{, 3} \otimes e_{3} .
\end{aligned}
$$

Because we intend to use the frame invariance of the energy, we will need the product of $\nabla_{h} y^{h}$ with $R_{\varepsilon}^{\top}=$ $I+\mathcal{O}(\varepsilon)$. First we have:

$$
\varepsilon h R_{\varepsilon}^{\top} A^{h}=\varepsilon h A^{h}+o(\varepsilon h)=\varepsilon h A^{h},
$$

where we have subsumed terms $o(\varepsilon h)$ into the $o(1)$ inside $A^{h}$. Therefore

$$
R_{\varepsilon}^{\top} \nabla_{h} y^{h}=I_{3}+\varepsilon h A^{h} \underbrace{-h e_{3} \otimes \hat{\nabla} \alpha+R_{\varepsilon}^{\top} h D_{, 3} \otimes e_{3}}_{=: h F^{h}} .
$$

Step 3: Convergence of the energies. The next step is a Taylor expansion around the identity. Given that the energy is scaled by $(\varepsilon h)^{-2}$, only those terms scaling as $\varepsilon h$ in (5.4) will remain: anything beyond that will not be seen and anything below will make the energy blow up. This means that we must choose $D$ so that

$$
\begin{aligned}
F^{h}= & D_{, 3} \otimes e_{3}+\varepsilon\left(v_{, 1} D_{3,3}, v_{, 2} D_{3,3},-v_{, 1} D_{1,3}-v_{, 2} D_{2,3}\right) \otimes e_{3} \\
& -e_{3} \otimes \hat{\nabla} \alpha+o(\varepsilon) \\
= & o(\varepsilon)
\end{aligned}
$$

Although these equations have no solution the symmetrised version does, ${ }^{13}$ so that for every smooth choice of $\alpha$ we can pick a bounded $D$ such that

$$
F_{s}^{h}=0, \text { and } F^{h}=\mathcal{O}(1)
$$

a fact that we will exploit next. By frame invariance and (5.4), we can write

$$
\begin{aligned}
W_{0}\left(x_{3}, \nabla_{h} y^{h}\left(I+\varepsilon h B^{h}\right)\right) & =W_{0}\left(x_{3}, R_{\varepsilon}^{\top} \nabla_{h} y^{h}\left(I+\varepsilon h B^{h}\right)\right) \\
& =W_{0}\left(x_{3},\left(I+\varepsilon h A^{h}+h F^{h}\right)\left(I+\varepsilon h B^{h}\right)\right) \\
& =W_{0}(x_{3}, I+h \underbrace{\left(\varepsilon\left(A^{h}+B^{h}\right)+F^{h}+o(\varepsilon)\right)}_{=: C^{h}}) .
\end{aligned}
$$

\footnotetext{
${ }^{13}$ Dividing by $h$ we arrive at:

$$
\left\{\begin{aligned}
D_{1,3}+\varepsilon v_{, 1} D_{3,3} & =\alpha, 1+o(\varepsilon), \\
D_{2,3}+\varepsilon v_{, 2} D_{3,3} & =\alpha, 2+o(\varepsilon), \\
D_{3,3}-\varepsilon v_{, 1} D_{1,3}-\varepsilon v_{, 2} D_{2,3} & =o(\varepsilon),
\end{aligned}\right.
$$
}

with solution:

$$
D\left(x^{\prime}, x_{3}\right)=x_{3} \hat{\nabla} \alpha+x_{3} \varepsilon \nabla v \cdot \nabla \alpha e_{3} .
$$


Because of (5.5) by our choice of $D$ we need to subtract the antisymmetric part of $F^{h}$, which we do by means of another rotation and frame invariance:

$$
\begin{aligned}
W_{0}\left(x_{3}, I+h C^{h}\right) & =W_{0}\left(x_{3}, \mathrm{e}^{-h F_{a}^{h}}\left(I+h C^{h}\right)\right) \\
& =W_{0}\left(x_{3}, I+h C^{h}-h F_{a}^{h}+\mathcal{O}\left(h^{2}\right)\right) \\
& =W_{0}\left(x_{3}, I+\varepsilon h\left(A^{h}+B^{h}\right)+o(\varepsilon h)\right) .
\end{aligned}
$$

Now whenever $h$ is small enough that $I+h C^{h}$ belongs to the neighbourhood of $\mathrm{SO}(3)$ where $W_{0}$ is twice differentiable, we can apply Taylor's theorem and the fact that $Q_{3}$ vanishes on antisymmetric matrices to see that, as $h \rightarrow 0$ :

$$
\begin{aligned}
\frac{1}{\varepsilon^{2} h^{2}} W_{0}\left(x_{3}, \nabla_{h} y^{h}\left(I+\varepsilon h B^{h}\right)\right) & =\frac{1}{2} Q_{3}\left(x_{3},\left(A^{h}+B^{h}\right)_{s}\right)+o(1) \\
& \rightarrow \frac{1}{2} Q_{3}\left(x_{3}, A_{s}+B_{s}\right),
\end{aligned}
$$

where

$$
A_{s}=\left(\alpha-x_{3}\right) \hat{\nabla}^{2} v+\hat{\nabla}_{s} g+\left(d \otimes e_{3}\right)_{s}
$$

We choose

$$
d\left(x^{\prime}, x_{3}\right)=\mathcal{L}\left(x_{3},\left(\alpha-x_{3}\right) \nabla^{2} v+\nabla_{s} g+\check{B}_{s}\right)-B_{\cdot 3},
$$

with $\mathcal{L}$ the map from (2.3), which by (2.3) and (2.4) is linear in the second component and satisfies $|\mathcal{L}(t, A)| \lesssim|A|$ uniformly in $t$, and $B .3$ the third column of $B$. Because the matrix $\left(\alpha-x_{3}\right) \nabla^{2} v+\nabla_{s} g+\check{B}_{s}$ is bounded uniformly in $x^{\prime}$, by the bound (2.4) the map

$$
x \mapsto \int_{0}^{x_{3}} \mathcal{L}\left(\xi,(\alpha-\xi) \hat{\nabla}^{2} v+\hat{\nabla}_{s} g+B_{s}(\xi)\right) \mathrm{d} \xi,
$$

is in $W^{1, \infty}\left(\Omega_{1} ; \mathbb{R}^{3}\right)$ and $y^{h} \in W^{1,2}$ as required (for the derivatives with respect to $x^{\prime}$ note that $v, g$ are smooth and $B$ independent of $\left.x^{\prime}\right)$.

Now, all quantities being bounded, by dominated convergence:

$$
\begin{aligned}
\mathcal{I}_{\alpha}^{h}\left(y^{h}\right) & \rightarrow \frac{1}{2} \int_{\Omega_{1}} Q_{3}\left(x_{3},\left(\alpha-x_{3}\right) \hat{\nabla}^{2} v+\hat{\nabla}_{s} g+\left(d \otimes e_{3}\right)_{s}+B_{s}\right) \\
& =\frac{1}{2} \int_{\Omega_{1}} Q_{2}\left(x_{3},\left(\alpha-x_{3}\right) \nabla^{2} v+\nabla_{s} g+\check{B}_{s}\right) .
\end{aligned}
$$

Note that a final step is required to obtain convergence to $\mathcal{I}_{1 \mathrm{Ki}}(v)$.

Step 4: Convergence of the deformations: $P_{\alpha}^{h}\left(y^{h}\right) \rightarrow v$ in $W^{1,2}$. Choose $R^{h} \equiv I \in \mathrm{SO}(3), c^{h} \equiv 0 \in \mathbb{R}^{3}$ in the definition of $\rho$ for (2.8). We have

$$
P_{\alpha}^{h}\left(y^{h}\right)=\frac{1}{\varepsilon} \int_{-1 / 2}^{1 / 2} y_{3}^{h}\left(x^{\prime}, x_{3}\right) \mathrm{d} x_{3},
$$


where in (5.3) we defined $y_{3}^{h}\left(x^{\prime}, x_{3}\right)=\varepsilon v\left(x^{\prime}\right)+h\left(x_{3}-\alpha\left(x^{\prime}\right)\right) b_{\varepsilon 3}\left(x^{\prime}\right)+\mathcal{O}(\varepsilon h)$. Then:

$$
\begin{aligned}
\left|P_{\alpha}^{h}\left(y^{h}\right)-v\right|^{2} & =\left|\frac{1}{\varepsilon} \int_{-1 / 2}^{1 / 2}\left[\varepsilon v+h\left(x_{3}-\alpha\right) b_{\varepsilon 3}+\mathcal{O}(\varepsilon h)\right] \mathrm{d} x_{3}-v\right|^{2} \\
& =\mathcal{O}\left(\varepsilon^{-2} h^{2}\right)
\end{aligned}
$$

and consequently $\left\|P_{\alpha}^{h}\left(y^{h}\right)-v\right\|_{0,2} \rightarrow 0$. An analogous computation for the derivatives shows strong convergence in $W^{1,2}$.

Step 5: Simultaneous convergence. Finally, as in ([52], Thm. 3.2), in order for the energy to converge to the true limit, we must pick $\alpha$ and $g$ in (5.3) so as to approximate the minimum $\bar{Q}_{2}$. This is done with Corollary 7.4, substituting sequences of smooth functions $\left(\alpha_{k}\right)_{k \in \mathbb{N}},\left(g_{k}\right)_{k \in \mathbb{N}}$ for the functions $\alpha, g$. Then, for each fixed $k$ we have:

$$
\begin{aligned}
\mathcal{I}_{\alpha}^{h}\left(y_{k}^{h}\right) \underset{h \rightarrow 0}{\rightarrow} \frac{1}{2} \int_{\Omega_{1}} Q_{2}\left(x_{3},\left(\alpha_{k}-x_{3}\right) \nabla^{2} v+\nabla_{s} g_{k}+\check{B}_{s}\right) \\
=\frac{1}{2} \int_{\omega} \bar{Q}_{2}^{\star}\left(-\nabla^{2} v\right) \mathrm{d} x^{\prime}+o(1)_{k \rightarrow \infty},
\end{aligned}
$$

and

$$
\left\|P_{\alpha}^{h}\left(y_{k}^{h}\right)-v\right\|_{1,2}^{2} \leqslant C(k) \varepsilon^{-2} h^{2} .
$$

And by a diagonal argument we can find $\left(y^{h}\right)_{h>0}$ whose energy converges to $\mathcal{I}_{1 \mathrm{Ki}}(v)$ while maintaining the convergence of the deformations.

Remark 5.3. The recovery sequence defined in (5.3) is substantially different from the recovery sequence constructed in [23] for homogeneous layers. While the summand depending on $d$, which allows for relaxation of the strain in the thin film direction to account for the Poisson effect, is of a more complicated form, it still has a direct counterpart in the homogeneous case. The contributions involving $\alpha, g$ and $D$, however, are particular to heterogeneous layers. The mapping $\alpha$ is used to re-adjust the height $x_{3}=0$ to $x_{3}=\alpha\left(x^{\prime}\right)$ at which the film follows the isometry $\bar{y}_{\varepsilon}$ to leading order. Considering, e.g., a bilayer consisting of a soft material on top of a material with large moduli, one typically has $\alpha<0$. This, however, leads to new terms in the strain depending on $\nabla \alpha$. The last term involving $D$, in combination with the microrotation $\mathrm{e}^{-h F_{a}^{h}}$ introduced in the above proof, is used to compensate for these terms. Finally, the summand involving the in-plane term $g$, together with the strain induced by $\alpha b_{\varepsilon}$, by Corollary 7.4 allows for a full relaxation of the $x_{3}$-independent strain contributions and thus to pass to the relaxed quadratic form $\bar{Q}_{2}^{\star}$, which only depends of the linearized curvature term $\nabla^{2} v$.

Theorem 5.4 (Upper bound, von Kármán regime). Let $\alpha=3$ and consider displacements $(u, v) \in X_{\alpha=3}:=$ $W^{1,2}\left(\omega ; \mathbb{R}^{2}\right) \times W^{1,2}(\omega ; \mathbb{R})$. There exists a sequence $\left(y^{h}\right)_{h>0} \subset Y$ which $P_{\theta}^{h}$-converges to $(u, v)$ such that

$$
\lim _{h \rightarrow 0} \frac{1}{\theta} \mathcal{I}_{\alpha}^{h}\left(y^{h}\right)=\mathcal{I}_{\mathrm{vK}}^{\theta}(u, v),
$$

with $\mathcal{I}_{\mathrm{vK}}^{\theta}$ defined as in (3.2) by

$$
\mathcal{I}_{\mathrm{vK}}^{\theta}(u, v):=\frac{1}{2} \int_{\omega} \bar{Q}_{2}\left(\theta^{1 / 2}\left(\nabla_{s} u+\frac{1}{2} \nabla v \otimes \nabla v\right),-\nabla^{2} v\right)
$$

over $X_{\alpha=3}^{0}=W^{1,2}\left(\omega ; \mathbb{R}^{2}\right) \times W^{2,2}(\omega ; \mathbb{R})$ and as $\infty$ elsewhere. 
Proof. In order to build the recovery sequence $\left(y^{h}\right)_{h>0}$ we will use the map $\mathcal{L}:(-1 / 2,1 / 2) \times \mathbb{R}^{2 \times 2} \rightarrow \mathbb{R}^{3}$ given by (2.3), which for each $t$ realises the minimum of $Q_{3}\left(t, \hat{A}+c \otimes e_{3}\right), A \in \mathbb{R}^{2 \times 2}$, i.e.

$$
Q_{2}(t, A)=Q_{3}\left(t, \hat{A}+\mathcal{L}(t, A) \otimes e_{3}\right)=Q_{3}\left(t, \hat{A}+\left(\mathcal{L}(t, A) \otimes e_{3}\right)_{s}\right)
$$

where the last equality follows from the fact that $Q_{3}$ vanishes on antisymmetric matrices. Recall from (2.4) that $\mathcal{L}(t, \cdot)$ is linear for every $t$ and that $|\mathcal{L}(t, A)| \lesssim|A|$ uniformly in $t$.

The functional $\mathcal{I}_{\mathrm{vK}}^{\theta}$ is clearly continuous in $X_{\alpha}^{0}=W^{1,2}\left(\omega ; \mathbb{R}^{2}\right) \times W^{2,2}(\omega ; \mathbb{R})$ with the strong topologies, so a standard argument [7] shows that it is enough to consider $(u, v) \in C^{\infty}\left(\bar{\omega} ; \mathbb{R}^{2}\right) \times C^{\infty}(\bar{\omega} ; \mathbb{R})$, which is dense in $X_{\alpha}^{0}$. We define:

$$
\begin{aligned}
y^{h}\left(x^{\prime}, x_{3}\right):= & \left(\begin{array}{c}
x^{\prime} \\
h x_{3}
\end{array}\right)+\left(\begin{array}{c}
\theta h^{2} u\left(x^{\prime}\right) \\
\sqrt{\theta} h v\left(x^{\prime}\right)
\end{array}\right)-\sqrt{\theta} h^{2} x_{3}\left(\begin{array}{c}
\nabla v\left(x^{\prime}\right) \\
0
\end{array}\right) \\
& +\theta h^{3} d\left(x^{\prime}, x_{3}\right)
\end{aligned}
$$

where $d \in W^{1, \infty}\left(\Omega_{1} ; \mathbb{R}^{3}\right)$ is a vector field to be determined along the proof.

Step 1: Approximation of the energy. A direct computation yields

$$
\begin{aligned}
\nabla_{h} y^{h}= & I+\left(\begin{array}{c|c}
\theta h^{2} \nabla u & -h \sqrt{\theta} \nabla v \\
h \sqrt{\theta} \nabla^{\top} v & 0
\end{array}\right)-h^{2} \theta\left(\begin{array}{cc}
x_{3} \theta^{-1 / 2} \nabla^{2} v & 0 \\
0 & 0
\end{array}\right) \\
& +h^{2} \theta \partial_{3} d \otimes e_{3}+\mathcal{O}\left(h^{3}\right) \\
= & I+h \sqrt{\theta} \underbrace{\left(e_{3} \otimes \hat{\nabla} v-\hat{\nabla} v \otimes e_{3}\right)}_{E} \\
& +h^{2} \theta \underbrace{\left(\hat{\nabla} u-x_{3} \theta^{-1 / 2} \hat{\nabla}^{2} v+\partial_{3} d \otimes e_{3}\right)}_{F}+\mathcal{O}\left(h^{3}\right) .
\end{aligned}
$$

For later use we note here the product:

$$
\begin{aligned}
\nabla_{h}^{\top} y^{h} \nabla_{h} y^{h} & =\left(I+h \sqrt{\theta} E^{\top}+h^{2} \theta F^{\top}\right)\left(I+h \sqrt{\theta} E+h^{2} \theta F\right)+\mathcal{O}\left(h^{3}\right) \\
& =I+\underbrace{h \sqrt{\theta} 2 E_{s}}_{=0}+h^{2} \theta \underbrace{\left(2 F_{s}+E^{\top} E\right)}_{N}+\mathcal{O}\left(h^{3}\right),
\end{aligned}
$$

where we used that $E$ is antisymmetric. For any matrix $M$ with positive determinant we have the polar decomposition $M=U \sqrt{M^{\top} M}=U \sqrt{I+P}$, with $U \in \mathrm{SO}(3)$ and $P=M^{\top} M-I$. By the frame invariance of the energy and a Taylor expansion around the identity of the square root

$$
\begin{aligned}
W_{0}\left(x_{3}, M\right) & =W_{0}\left(x_{3}, \sqrt{M^{\top} M}\right) \\
& =W_{0}\left(x_{3}, I+\frac{1}{2}\left(M^{\top} M-I\right)+o\left(\left|M^{\top} M-I\right|\right)\right),
\end{aligned}
$$

and, assuming that a Taylor expansion of $W_{0}$ around the identity can be carried, i.e. that $M$ is close enough to $\mathrm{SO}(3)$, this is equal to:

$$
\frac{1}{2} Q_{3}\left(x_{3}, \frac{1}{2}\left(M^{\top} M-I\right)\right)+o\left(\left|M^{\top} M-I\right|^{2}\right)
$$


In view of the definition of $W_{0}$, we set

$$
M^{h}:=\nabla_{h} y^{h}\left(I+h^{2} \tilde{B}^{h}\right)
$$

where $\tilde{B}^{h}=\sqrt{\theta} B^{h} \rightarrow \tilde{B}=\sqrt{\theta} B$ in $L^{\infty}$. Then

$$
\begin{aligned}
\left(M^{h}\right)^{\top} M^{h} & :=\left[\nabla_{h} y^{h}\left(I+h^{2} \tilde{B}^{h}\right)\right]^{\top}\left[\nabla_{h} y^{h}\left(I+h^{2} \tilde{B}^{h}\right)\right] \\
& =\left(I+h^{2}\left(\tilde{B}^{h}\right)^{\top}\right)\left(I+h^{2} \theta N\right)\left(I+h^{2} \tilde{B}^{h}\right)+\mathcal{O}\left(h^{3}\right) \\
& =I+h^{2} \theta N+h^{2} 2 \tilde{B}_{s}+o\left(h^{2}\right) .
\end{aligned}
$$

To compute the first term in $h^{2}, N=2 F_{s}+E^{\top} E$, we have

$$
2 F_{s}=2\left(\hat{\nabla}_{s} u-x_{3} \theta^{-1 / 2} \hat{\nabla}^{2} v+\left(\partial_{3} d \otimes e_{3}\right)_{s}\right),
$$

and:

$$
\begin{aligned}
E^{\top} E & =\left(\hat{\nabla} v \otimes e_{3}-e_{3} \otimes \hat{\nabla} v\right)\left(e_{3} \otimes \hat{\nabla} v-\hat{\nabla} v \otimes e_{3}\right) \\
& =\hat{\nabla} v \otimes \hat{\nabla} v+|\hat{\nabla} v|^{2} e_{3} \otimes e_{3} .
\end{aligned}
$$

Since these quantities are independent of $h$, for sufficiently small $h$ the product $\left(M^{h}\right)^{\top} M^{h}$ does lie close enough to $\mathrm{SO}(3)$ and we can perform the desired Taylor expansion:

$$
\begin{aligned}
W^{h}\left(x_{3}, \nabla_{h} y^{h}\right) & =W_{0}\left(x_{3}, \nabla_{h} y^{h}\left(I+h^{2} \tilde{B}^{h}\right)\right) \\
& =W_{0}\left(x_{3},\left(\left(M^{h}\right)^{\top} M^{h}\right)^{1 / 2}\right) \\
& =\frac{1}{2} Q_{3}\left(x_{3}, \frac{1}{2}\left[\left(M^{h}\right)^{\top} M^{h}-I\right]\right)+o\left(\left|\left(M^{h}\right)^{\top} M^{h}-I\right|^{2}\right) .
\end{aligned}
$$

Define now $\hat{G}_{0}:=\theta\left(\hat{\nabla}_{s} u+\frac{1}{2} \hat{\nabla} v \otimes \hat{\nabla} v\right), \hat{G}_{1}:=-\theta^{1 / 2} \hat{\nabla}^{2} v$ as in Lemma 4.2. Bringing the previous computations together we obtain:

$$
\begin{aligned}
\frac{1}{2}\left[\left(M^{h}\right)^{\top} M^{h}-I\right]= & h^{2}\left[\hat{G}_{0}-x_{3} \hat{G}_{1}+\widehat{\tilde{\tilde{B}}}_{s}\right. \\
& +\underbrace{\sqrt{\theta}\left(B(t)_{\cdot 3} \otimes e_{3}\right)_{s}+\frac{\theta}{2}|\hat{\nabla} v|^{2} e_{3} \otimes e_{3}+\theta\left(\partial_{3} d \otimes e_{3}\right)_{s}}_{H}]
\end{aligned}
$$

hence

$$
\begin{aligned}
\frac{1}{h^{4}} & {\left[Q_{3}\left(x_{3}, \frac{1}{2}\left(\left(M^{h}\right)^{\top} M^{h}-I\right)\right)+o\left(\left|\left(M^{h}\right)^{\top} M^{h}-I\right|^{2}\right)\right] } \\
& =Q_{3}\left(x_{3}, \hat{G}_{0}-x_{3} \hat{G}_{1}+\sqrt{\theta} \widehat{\tilde{B}}_{s}+H\right)+o(1) .
\end{aligned}
$$

We now choose the vector field $d$ to cancel one term and attain the minimum for the others by solving for $\partial_{3} d$ in:

$$
H \stackrel{!}{=}\left(\mathcal{L}\left(x_{3}, G_{0}-x_{3} G_{1}+\sqrt{\theta} \check{B}_{s}\left(x_{3}\right)\right) \otimes e_{3}\right)_{s}
$$


that is:

$$
\theta^{-1 / 2} B(t)_{\cdot 3}+\frac{1}{2}|\hat{\nabla} v|^{2} e_{3}+\partial_{3} d\left(x^{\prime}, x_{3}\right)=\frac{1}{\theta} \mathcal{L}\left(x_{3}, G_{0}-x_{3} G_{1}+\sqrt{\theta} \check{B}_{s}\left(x_{3}\right)\right)
$$

Consequently, we set:

$$
\begin{aligned}
d\left(x^{\prime}, x_{3}\right):= & -\frac{1}{2}|\hat{\nabla} v|^{2} x_{3} e_{3} \\
& +\frac{1}{\theta} \int_{0}^{x_{3}} \mathcal{L}\left(t, G_{0}-t G_{1}+\sqrt{\theta} \check{B}_{s}(t)\right)-\sqrt{\theta} B(t)_{\cdot 3} \mathrm{~d} t
\end{aligned}
$$

and we obtain

$$
Q_{3}\left(x_{3}, \hat{G}_{0}-x_{3} \hat{G}_{1}+\sqrt{\theta} B_{s}\left(x_{3}\right)+H\right)=Q_{2}\left(x_{3}, G_{0}-x_{3} G_{1}+\sqrt{\theta} \check{B}_{s}\left(x_{3}\right)\right) .
$$

As in the proof of Theorem 5.2, (2.3) and (2.4) imply that $d \in W^{1, \infty}\left(\Omega_{1} ; \mathbb{R}^{3}\right)$.

Step 2: Convergence. By the previous step we have $\frac{1}{\theta h^{4}} W_{0}\left(x_{3}, \nabla_{h} y^{h}\right) \rightarrow \frac{1}{2 \theta} Q_{2}\left(x_{3}, G_{0}+x_{3} G_{1}+\sqrt{\theta} \check{B}_{s}\right)$ a.e. as $h \rightarrow 0$, and the sequence is uniformly bounded so we can integrate over the domain and pass to the limit:

$$
\begin{aligned}
\frac{1}{\theta h^{4}} \int_{\Omega_{1}} W^{h}\left(x_{3}, \nabla_{h} y^{h}\right) & \rightarrow \frac{1}{2 \theta} \int_{\Omega_{1}} Q_{2}\left(x_{3}, G_{0}-x_{3} G_{1}+\sqrt{\theta} \check{B}_{s}\right) \\
& =\frac{1}{2} \int_{\omega} \bar{Q}_{2}\left(\theta^{1 / 2}\left(\nabla_{s} u+\frac{1}{2} \nabla v \otimes \nabla v\right),-\nabla^{2} v\right) .
\end{aligned}
$$

Step 3: Convergence of the recovery sequence. Note that $P_{\theta}^{h}\left(y^{h}\right) \rightarrow(u, v)$ in $X_{\alpha}$ as $h \rightarrow 0$ with the choice $R^{h}=$ $I \in \mathrm{SO}(3), c^{h}=0 \in \mathbb{R}^{3}$ in Definition 2.2 since

$$
\begin{gathered}
\frac{1}{\theta h^{2}} \int_{-1 / 2}^{1 / 2}\left(y^{h}\left(\cdot, x_{3}\right)-x^{\prime}\right) \mathrm{d} x_{3} \longrightarrow u \quad \text { in } W^{1,2}\left(\omega ; \mathbb{R}^{2}\right), \\
\quad \frac{1}{\sqrt{\theta} h} \int_{-1 / 2}^{1 / 2} y_{3}^{h}\left(\cdot, x_{3}\right) \mathrm{d} x_{3} \longrightarrow v \quad \text { in } W^{1,2}(\omega ; \mathbb{R}) .
\end{gathered}
$$

In the next result, there is a departure from the analogous functional in [23] beyond the dependence on the out-of-plane component $x_{3}$. In the preceding cases, if one sets $Q_{2}(t, A) \equiv Q_{2}(A)$, and $B \equiv 0$ then the same functionals are obtained as in that work. However, in the regime $\alpha>3$ their limit has no membrane term, but we have $\bar{Q}_{2}\left(\nabla_{s} u,-\nabla^{2} v\right)=\frac{1}{2} \int Q_{2}\left(\nabla_{s} u\right)+\frac{1}{24} \int Q_{2}\left(\nabla^{2} v\right)$, with the membrane term. The reason is that [23] discard the in-plane displacements by minimising them away. In their proofs, they drop the first term in the lower bound and build the recovery sequence with no $u$ term in $h^{\alpha-1}$.

Note that it is by keeping the membrane term that our model is able to take into account and respond to the pre-stressing (internal misfit) $B^{h}$, e.g. compressive or tensile stresses in wafers.

Theorem 5.5 (Upper bound, linearised von Kármán regime). Let $\alpha>3$ and consider displacements ( $u, v) \in$ $X_{\alpha}:=W^{1,2}\left(\omega ; \mathbb{R}^{2}\right) \times W^{1,2}(\omega ; \mathbb{R})$. There exists a sequence $\left(y^{h}\right)_{h>0} \subset Y$ which $P_{\alpha}^{h}$-converges to $(u, v)$ such that

$$
\lim _{h \rightarrow 0} \mathcal{I}_{\alpha}^{h}\left(y^{h}\right)=\mathcal{I}_{\mathrm{lvK}}(u, v)
$$


with $\mathcal{I}_{\mathrm{lvK}}$ defined as in (3.3) by

$$
\mathcal{I}_{\mathrm{lvK}}(u, v):=\frac{1}{2} \int_{\omega} \bar{Q}_{2}\left(\nabla_{s} u,-\nabla^{2} v\right) \mathrm{d} x^{\prime}
$$

on $X_{\alpha}^{0}$ and by $+\infty$ elsewhere.

Proof. We follow closely the notation and path of proof of Theorem 5.4. By a standard density argument it is enough to consider $(u, v) \in X_{\alpha} \cap C^{\infty}(\bar{\omega})$. Define

$$
y^{h}\left(x^{\prime}, x_{3}\right):=\left(\begin{array}{c}
x^{\prime} \\
h x_{3}
\end{array}\right)+\left(\begin{array}{c}
h^{\alpha-1} u\left(x^{\prime}\right) \\
h^{\alpha-2} v\left(x^{\prime}\right)
\end{array}\right)-h^{\alpha-1} x_{3}\left(\begin{array}{c}
\nabla v\left(x^{\prime}\right) \\
0
\end{array}\right)+h^{\alpha} d\left(x^{\prime}, x_{3}\right),
$$

with $d \in W^{1, \infty}\left(\Omega_{1} ; \mathbb{R}^{3}\right)$. Then

$$
\begin{aligned}
\nabla_{h} y^{h}= & I+h^{\alpha-2} \underbrace{\left(e_{3} \otimes \hat{\nabla} v-\hat{\nabla} v \otimes e_{3}\right)}_{=: E}+h^{\alpha-1} \underbrace{\left(\hat{\nabla} u-x_{3} \hat{\nabla}^{2} v+\partial_{3} d \otimes e_{3}\right)}_{=: F} \\
& +\mathcal{O}\left(h^{\alpha}\right),
\end{aligned}
$$

and, using that $E_{s}=0$ :

$$
\begin{aligned}
\nabla_{h}^{\top} y^{h} \nabla_{h} y^{h} & =\left(I+h^{\alpha-2} E^{\top}+h^{\alpha-1} F^{\top}\right)\left(I+h^{\alpha-2} E+h^{\alpha-1} F\right)+\mathcal{O}\left(h^{\alpha}\right) \\
& =I+2 h^{\alpha-1} F_{s}+o\left(h^{\alpha-1}\right) .
\end{aligned}
$$

Define now $M^{h}:=\nabla_{h} y^{h}\left(I+h^{\alpha-1} B^{h}\right)$. A few computations lead to

$$
\frac{1}{2}\left[\left(M^{h}\right)^{\top} M^{h}-I\right]=h^{\alpha-1}\left(F_{s}+B_{s}\right)+o\left(h^{\alpha-1}\right),
$$

from which follows, after a Taylor approximation (recall from the proof of Theorem 5.4, that this can be done for sufficiently small $h$ ):

$$
\begin{aligned}
\frac{1}{h^{2 \alpha-2}} W^{h}\left(x_{3}, \nabla_{h} y^{h}\right)= & \frac{1}{2 h^{2 \alpha-2}}\left[Q_{3}\left(x_{3},\left[\left(M^{h}\right)^{\top} M^{h}-I\right] / 2\right)\right. \\
& \left.+o\left(\left|\left(M^{h}\right)^{\top} M^{h}-I\right|^{2}\right)\right] \\
= & \frac{1}{2} Q_{3}\left(x_{3}, F_{s}+B_{s}\right)+o(1) .
\end{aligned}
$$

Picking $d$ such that:

$$
\left(\left(B\left(x_{3}\right)_{\cdot 3}+\partial_{3} d\right) \otimes e_{3}\right)_{s}=\left(\mathcal{L}\left(x_{3}, \nabla u-x_{3} \nabla^{2} v+\check{B}_{s}\left(x_{3}\right)\right) \otimes e_{3}\right)_{s}
$$

e.g.

$$
d\left(x^{\prime}, x_{3}\right):=\int_{0}^{x_{3}} \mathcal{L}\left(t, \nabla_{s} u-t \nabla^{2} v+\check{B}_{s}(t)\right)-B(t) ._{\cdot 3} \mathrm{~d} t
$$

the term with $\mathcal{L}$ in $Q_{2}$ cancels out and we obtain

$$
Q_{3}\left(x_{3}, F_{s}+B_{s}\right)=Q_{2}\left(x_{3}, \nabla_{s} u-x_{3} \nabla^{2} v+\check{B}_{s}\left(x_{3}\right)\right) .
$$


Note that as proved in Theorem 5.4, the properties of $\mathcal{L}$ imply that the function $d \in W^{1, \infty}\left(\Omega_{1} ; \mathbb{R}^{3}\right)$ so the previous computations are justified. We have therefore

$$
\frac{1}{h^{2 \alpha-2}} W_{0}\left(x_{3}, \nabla_{h} y^{h}\right) \rightarrow \frac{1}{2} Q_{2}\left(x_{3}, \nabla_{s} u-x_{3} \nabla^{2} v+\check{B}_{s}\left(x_{3}\right)\right) \text { a.e. in } \omega,
$$

and also $Q_{2}\left(x_{3}, A\right) \lesssim|A|^{2}$ by Assumption 2.1.b. Because $u_{i}, v \in C^{\infty}(\bar{\omega})$ and $B_{s} \in L^{\infty}$, all arguments of $Q_{2}$ are uniformly bounded and we can apply dominated convergence to conclude:

$$
\begin{aligned}
\frac{1}{h^{2 \alpha-2}} \int_{\Omega_{1}} W_{0}\left(x_{3}, \nabla_{h} y^{h}\right) & \underset{h \downarrow 0}{\longrightarrow} \frac{1}{2} \int_{\Omega_{1}} Q_{2}\left(x_{3}, \nabla_{s} u-x_{3} \nabla^{2} v+\check{B}_{s}\left(x_{3}\right)\right) \mathrm{d} x \\
& =\frac{1}{2} \int_{\omega} \bar{Q}_{2}\left(\nabla_{s} u,-\nabla^{2} v\right) \mathrm{d} x^{\prime}
\end{aligned}
$$

Set now $R=I \in \mathrm{SO}(3), c=0 \in \mathbb{R}^{3}$ for the rigid transformation $\rho$ in Definition 2.2. It remains to note that indeed $P_{\alpha}^{h}\left(y^{h}\right) \rightarrow(u, v)$ in $X_{\alpha}$ :

$$
\begin{aligned}
& \frac{1}{h^{\alpha-2}} \int_{-1 / 2}^{1 / 2} y_{3}^{h}\left(\cdot, x_{3}\right) \mathrm{d} x_{3} \longrightarrow v \quad \text { in } W^{1,2}(\omega ; \mathbb{R}), \\
& \frac{1}{h^{\alpha-1}} \int_{-1 / 2}^{1 / 2}\left(y^{h \prime}\left(\cdot, x_{3}\right)-x^{\prime}\right) \mathrm{d} x_{3} \longrightarrow u \quad \text { in } W^{1,2}\left(\omega ; \mathbb{R}^{2}\right),
\end{aligned}
$$

and the proof is complete.

\section{6. Г-CONVERGENCE OF THE INTERPOlATING THEORY}

Notation. Throughout this section we write $A_{\theta}:=\nabla_{s} u_{\theta}+\frac{1}{2} \nabla v_{\theta} \otimes \nabla v_{\theta}$ for the strain induced by a pair of displacements $\left(u_{\theta}, v_{\theta}\right)$. As before, $\theta>0$.

We now set to prove Theorem 3.3, which states that the functional of generalised von Kármán type that we found in the preceding section,

$$
\mathcal{I}_{\mathrm{vK}}^{\theta}\left(u_{\theta}, v_{\theta}\right):=\frac{1}{2} \int_{\omega} \int_{-1 / 2}^{1 / 2} Q_{2}\left(x_{3}, \sqrt{\theta} A_{\theta}-x_{3} \nabla^{2} v_{\theta}+\check{B}\left(x_{3}\right)\right) \mathrm{d} x_{3} \mathrm{~d} x^{\prime},
$$

interpolates between the two adjacent regimes as $\theta \rightarrow \infty$ or $\theta \rightarrow 0$. As $\theta$ approaches infinity, we expect the optimal energy configurations to approach those of the linearised Kirchhoff model, whereas with $\theta$ tending to zero they should approach the linearised von Kármán model.

For this section we restrict ourselves to spaces where Korn-Poincaré type inequalities hold.

Definition 6.1. Let

$$
X_{u}:=\left\{u \in W^{1,2}\left(\omega ; \mathbb{R}^{2}\right): \int_{\omega} \nabla_{a} u=0 \text { and } \int_{\omega} u=0\right\}
$$

and

$$
X_{v}:=\left\{v \in W^{2,2}(\omega ; \mathbb{R}): \int_{\omega} \nabla v=0 \text { and } \int_{\omega} v=0\right\}
$$


We set $X_{w}:=X_{u} \times X_{v}$ with the weak topologies.

Additionally, from now on we assume without loss that the barycenter of $\omega$ be the origin so that $\int_{\omega} x^{\prime} \mathrm{d} x^{\prime}=0$. Finally, for the $\Gamma$-limit $\theta \rightarrow \infty$ we require that $\omega$ be convex or simply connected with $\partial \omega$ satisfying the condition in Remark 3.2.4 and recall the definition of the space of maps with singular Hessian

$$
W_{s h}^{2,2}(\omega):=\left\{v \in W^{2,2}(\omega ; \mathbb{R}): \operatorname{det} \nabla^{2} v=0 \text { a.e. }\right\}
$$

Remark 6.2. There is no loss of generality in reducing to the space $X_{u} \times X_{v}$ : First we can always add an infinitesimal rigid motion to $u$ and any affine function to $v$ without changing $\nabla_{s} u$ or $\nabla^{2} v$. Second, although the nonlinear term $\nabla v \otimes \nabla v$ does change after adding an affine function, the extra terms appearing happen to be a symmetric gradient which can be absorbed into $\nabla_{s} u$ with a little help: For any $g(x)=a \cdot x+b$ for $a, b \in \mathbb{R}^{2}$, we have

$$
\begin{aligned}
\nabla(v+g) \otimes \nabla(v+g) & =\nabla v \otimes \nabla v+a \otimes a+a \otimes \nabla v+\nabla v \otimes a \\
& =\nabla v \otimes \nabla v+\nabla_{s} z
\end{aligned}
$$

where we set $z(x):=(2 v(x)+a \cdot x) a \in W^{2,2}\left(\omega ; \mathbb{R}^{2}\right)$. Therefore, for any fixed $u \in W^{1,2}\left(\omega ; \mathbb{R}^{2}\right), v \in W^{2,2}(\omega)$ one can choose $g(x)=-\left[(\nabla v)_{\omega} \cdot x+(v)_{\omega}\right]$ and define

$$
\tilde{u}=u+z+r, \quad \tilde{v}=v+g,
$$

with $r(x)=R x+c$, for constants $R:=\frac{-1}{|\omega|} \int_{\omega} \nabla_{a} u+\nabla_{a} z \mathrm{~d} x \in \mathbb{R}_{\text {ant }}^{2 \times 2}$ and $c:=\frac{-1}{|\omega|} \int_{\omega} u(x)+z(x)+R x \mathrm{~d} x$. For $\tilde{u}, \tilde{v}$ we then have on the one hand $\int \tilde{u}=0, \int \nabla_{a} \tilde{u}=0$ and $\int \tilde{v}=0, \int \nabla \tilde{v}=0$ and on the other (note that $\nabla_{s} r=0$ ):

$$
\mathcal{I}_{\mathrm{vK}}^{\theta}(u, v)=\mathcal{I}_{\mathrm{vK}}^{\theta}(\tilde{u}-z-r, \tilde{v}-g) \stackrel{(6.1)}{=} \mathcal{I}_{\mathrm{vK}}^{\theta}(\tilde{u}-r, \tilde{v})=\mathcal{I}_{\mathrm{vK}}^{\theta}(\tilde{u}, \tilde{v})
$$

as desired.

Our first theorem identifies the types of convergence required in order to obtain precompactness of sequences of bounded energy. We use these definitions of convergence for the computation of the $\Gamma$-limits.

Theorem 6.3 (Compactness). Let $\left(u_{\theta}, v_{\theta}\right)_{\theta>0}$ be a sequence in $X_{w}$ with finite energy

$$
\sup _{\theta>0}^{\theta} \mathcal{I}_{\mathrm{vK}}\left(u_{\theta}, v_{\theta}\right) \leqslant C .
$$

Then:

1. The sequence $\left(v_{\theta}\right)_{\theta \uparrow \infty}$ is weakly precompact in $W^{2,2}(\omega)$ and the weak limit is in $X_{v} \cap W_{s h}^{2,2}(\omega)$. Additionally $\left(u_{\theta}\right)_{\theta \uparrow \infty}$ is weakly precompact in $W^{1,2}\left(\omega ; \mathbb{R}^{2}\right)$.

2. The sequence $\left(\theta^{1 / 2} u_{\theta}, v_{\theta}\right)_{\theta \downarrow 0}$ is weakly precompact in $W^{1,2}\left(\omega ; \mathbb{R}^{2}\right) \times W^{2,2}(\omega)$ and the weak limit is in $X_{u} \times X_{v}$.

Proof. By assumption:

$$
C \geqslant \int_{\omega} \int_{-1 / 2}^{1 / 2} Q_{2}\left(x_{3}, \sqrt{\theta} A_{\theta}-x_{3} \nabla^{2} v_{\theta}+\check{B}\left(x_{3}\right)\right) \mathrm{d} x_{3} \mathrm{~d} x^{\prime}
$$

and the uniform lower bound on $Q_{2}$ in (2.4) yields

$$
Q_{2}\left(x_{3}, F\right) \gtrsim|F|^{2} \text { for all symmetric } F \text { and } x_{3} \in(-1 / 2,1 / 2),
$$


so that $\int_{-1 / 2}^{1 / 2} Q_{2}\left(x_{3}, F\left(x_{3}\right)\right) \gtrsim \int_{-1 / 2}^{1 / 2}\left|F\left(x_{3}\right)\right|^{2}$. Now split the inner integral in half, and normalise to use Jensen's inequality. In the upper half:

$$
\begin{aligned}
C & \gtrsim \int_{\omega} 2 \int_{0}^{1 / 2}\left|\sqrt{\theta} A_{\theta}-x_{3} \nabla^{2} v_{\theta}+\check{B}_{s}\left(x_{3}\right)\right|^{2} \mathrm{~d} x_{3} \mathrm{~d} x^{\prime} \\
& \gtrsim \int_{\omega}\left|2 \int_{0}^{1 / 2} \sqrt{\theta} A_{\theta}-x_{3} \nabla^{2} v_{\theta}+\check{B}_{s}\left(x_{3}\right) \mathrm{d} x_{3}\right|^{2} \mathrm{~d} x^{\prime} \\
& =\int_{\omega}\left|\sqrt{\theta} A_{\theta}-\frac{1}{4} \nabla^{2} v_{\theta}+c\right|^{2} \mathrm{~d} x^{\prime} \\
& \gtrsim\left\|\sqrt{\theta} A_{\theta}-\frac{1}{4} \nabla^{2} v_{\theta}\right\|_{0,2}^{2}-c^{2}|\omega| .
\end{aligned}
$$

An analogous computation for the lower half of the interval results in

$$
C \geqslant\left\|\sqrt{\theta} A_{\theta}+\frac{1}{4} \nabla^{2} v_{\theta}\right\|_{0,2}
$$

and bringing both bounds together we obtain:

$$
\left\|\sqrt{\theta} A_{\theta}\right\|_{0,2} \leqslant C \text { and }\left\|\nabla^{2} v_{\theta}\right\|_{0,2} \leqslant C .
$$

Two applications of Poincaré's inequality to the second bound yield:

$$
\left\|v_{\theta}\right\|_{2,2} \leqslant C \text { for all } \theta>0 .
$$

Therefore a subsequence (not relabelled) $v_{\theta} \rightarrow v$ for some $v \in X_{v}$. Now consider (6.2) again and observe that with the Sobolev embedding $W^{1,2}(\omega) \hookrightarrow L^{4}(\omega)$ we know that

$$
\left\|\nabla v_{\theta} \otimes \nabla v_{\theta}\right\|_{0,2}=\left\|\nabla v_{\theta}\right\|_{0,4}^{2} \lesssim\left\|\nabla v_{\theta}\right\|_{1,2}^{2} \leqslant\left\|v_{\theta}\right\|_{2,2}^{2} \leqslant C .
$$

Together with (6.2) this implies

$$
\left\|\sqrt{\theta} \nabla_{s} u_{\theta}\right\|_{0,2} \leqslant C+C \sqrt{\theta}
$$

so, by the Korn-Poincaré inequality, the sequence $\left(u_{\theta}\right)_{\theta>0}$ is bounded in $W^{1,2}$ when $\theta \rightarrow \infty$ and there exists a subsequence (not relabelled) $u_{\theta} \rightarrow u$ for some $u \in X_{u}$.

Now if $z_{\varepsilon} \rightarrow z$ in $W^{1,2}\left(\omega ; \mathbb{R}^{2}\right)$, by the compact Sobolev embedding $W^{1,2} \hookrightarrow L^{4}$ we have $z_{\varepsilon} \rightarrow z$ in $L^{4}$ and

$$
\int_{\omega}\left|z_{\varepsilon} \otimes z_{\varepsilon}-z \otimes z\right|^{2} \mathrm{~d} x \underset{\varepsilon \rightarrow 0}{\longrightarrow} 0
$$

So $\nabla v_{\theta} \otimes \nabla v_{\theta} \rightarrow \nabla v \otimes \nabla v$ in $L^{2}$ and from (6.2) and lower semicontinuity of the norm we deduce

$$
\left\|\nabla_{s} u+\frac{1}{2} \nabla v \otimes \nabla v\right\|_{0,2} \leqslant \operatorname{linf}_{\theta \rightarrow \infty}\left\|A_{\theta}\right\|_{0,2}=0 .
$$

([23], Prop. 9) (applied to every ball contained in $\omega$ ) shows $\operatorname{det} \nabla^{2} v=0$ a.e., and this concludes the proof of the first statement. 
For the second statement we take $\theta \downarrow 0$. It only remains to prove precompactness for $u_{\theta}$ since the previous computation for $\left(v_{\theta}\right)_{\theta>0}$ applies for all $\theta$. But it follows directly from (6.3) above: again with the Korn-Poincaré inequality, the sequence $\left(\theta^{1 / 2} u_{\theta}\right)_{\theta>0}$ is bounded in $W^{1,2}$, so it contains a weakly convergent subsequence $\theta^{1 / 2} u_{\theta} \rightarrow u \in X_{u}$.

We begin the proof of $\Gamma$-convergence in Theorem 3.3 with the lower and upper bound and a few technical lemmas for the passage from $\alpha=3$ to $\alpha<3$.

Theorem 6.4 (Lower bound, von Kármán to linearised Kirchhoff). Assume $\omega$ is convex and let $\left(u_{\theta}, v_{\theta}\right)_{\theta>0}$ be a sequence in $X_{w}$ such that $v_{\theta} \rightarrow v$ in $X_{v}$ as $\theta \rightarrow \infty$. Then

$$
\operatorname{linf}_{\theta \uparrow \infty} \mathcal{I}_{\mathrm{vK}}^{\theta}\left(u_{\theta}, v_{\theta}\right) \geqslant \mathcal{I}_{\mathrm{lKi}}(v) .
$$

Proof. By Theorem 6.3 we only need to consider $v \in X_{v}^{0}:=X_{v} \cap W_{s h}^{2,2}(\omega)$, hence $\mathcal{I}_{1 \mathrm{Ki}}(v)<\infty$. We can minimise the inner integral pointwise and obtain a lower bound:

$$
\begin{aligned}
\mathcal{I}_{\mathrm{vK}}^{\theta}\left(u_{\theta}, v_{\theta}\right) & =\frac{1}{2} \int_{\omega} \int_{-1 / 2}^{1 / 2} Q_{2}\left(x_{3}, \sqrt{\theta} A_{\theta}-x_{3} \nabla^{2} v_{\theta}+\check{B}\left(x_{3}\right)\right) \mathrm{d} x_{3} \mathrm{~d} x^{\prime} \\
& \geqslant \frac{1}{2} \int_{\omega} \min _{A \in \mathbb{R}^{2 \times 2}} \int_{-1 / 2}^{1 / 2} Q_{2}\left(x_{3}, A-x_{3} \nabla^{2} v_{\theta}+\check{B}\left(x_{3}\right)\right) \mathrm{d} x_{3} \mathrm{~d} x^{\prime} \\
& =\mathcal{I}_{1 \mathrm{Ki}}\left(v_{\theta}\right) .
\end{aligned}
$$

As $\bar{Q}_{2}^{\star}$ is a convex quadratic form, we have by the convergence $\nabla^{2} v_{\theta} \rightarrow \nabla^{2} v$ in $L^{2}$ :

$$
\operatorname{linf}_{\theta \uparrow \infty} \mathcal{I}_{\mathrm{vK}}^{\theta}\left(u_{\theta}, v_{\theta}\right) \geqslant \operatorname{linf}_{\theta \uparrow \infty} \mathcal{I}_{\mathrm{lKi}}\left(v_{\theta}\right) \geqslant \mathcal{I}_{\mathrm{lKi}}(v)
$$

Theorem 6.5 (Upper bound, von Kármán to linearised Kirchhoff). Assume $\omega$ is convex ${ }^{14}$ and fix some $v \in X_{v}$. There exists a sequence $\left(u_{\theta}, v_{\theta}\right)_{\theta \uparrow \infty} \subset X_{w}$ such that $v_{\theta} \rightarrow v$ in $W^{2,2}(\omega)$ and $\mathcal{I}_{\mathrm{vK}}^{\theta}\left(u_{\theta}, v_{\theta}\right) \rightarrow \mathcal{I}_{1 \mathrm{Ki}}(v)$ as $\theta \rightarrow \infty$.

Proof. Without loss of generality we may assume that $v \in X_{v}^{0}:=X_{v} \cap W_{s h}^{2,2}(\omega)$. By Theorem 7.1 we can work with functions $v \in \mathcal{V}_{0}$, see (7.2), which are smooth with singular Hessian, since they are dense in the restriction to $X_{v}^{0}$. By ([23], Prop. 9) there exists a displacement $u: \omega \rightarrow \mathbb{R}^{2}$ in $W^{2,2}\left(\omega ; \mathbb{R}^{2}\right)$ such that

$$
\nabla_{s} u+\frac{1}{2} \nabla v \otimes \nabla v=0
$$

Fix $\delta>0$ and, using Corollary 7.4, choose smooth functions $\alpha \in C^{\infty}(\bar{\omega}), g \in C^{\infty}\left(\bar{\omega} ; \mathbb{R}^{2}\right)$ such that

$$
\left\|\nabla_{s} g+\alpha \nabla^{2} v-A_{\min }\right\|_{0,2}^{2}<\delta
$$

where $A_{\min } \in L^{\infty}\left(\omega ; \mathbb{R}_{\mathrm{sym}}^{2 \times 2}\right)$ is defined as

$$
A_{\min }:=\underset{A \in \mathbb{R}_{\mathrm{sym}}^{2 \times 2}}{\operatorname{argmin}} \int_{-1 / 2}^{1 / 2} Q_{2}\left(t, A-t \nabla^{2} v+\check{B}(t)\right) \mathrm{d} t .
$$

\footnotetext{
${ }^{14}$ Alternatively we may assume that $\omega$ be simply connected and $\partial \omega$ satisfy the assumption detailed in Remark 3.2 .4 , because Theorem 7.1, Corollary 7.4 and ([23], Prop. 9) also apply in this situation.
} 
Define now the recovery sequence $\left(u_{\theta}, v_{\theta}\right)_{\theta>0}$ with

$$
u_{\theta}:=u+\frac{1}{\sqrt{\theta}}(\alpha \nabla v+g), \quad v_{\theta}:=v-\frac{1}{\sqrt{\theta}} \alpha .
$$

Clearly $v_{\theta}=v-\theta^{-1 / 2} \alpha \rightarrow v$ as $\theta \rightarrow \infty$ in $W^{2,2}(\omega)$. Furthermore

$$
\begin{gathered}
\sqrt{\theta} \nabla_{s} u_{\theta}=\sqrt{\theta} \nabla_{s} u+\nabla_{s} g+(\nabla \alpha \otimes \nabla v)_{s}+\alpha \nabla^{2} v \\
\frac{\sqrt{\theta}}{2} \nabla v_{\theta} \otimes \nabla v_{\theta}=\frac{\sqrt{\theta}}{2} \nabla v \otimes \nabla v+\frac{1}{2 \sqrt{\theta}} \nabla \alpha \otimes \nabla \alpha-(\nabla \alpha \otimes \nabla v)_{s},
\end{gathered}
$$

and

$$
-t \nabla^{2} v_{\theta}=-t \nabla^{2} v+\frac{t}{\sqrt{\theta}} \nabla^{2} \alpha
$$

so that, using (6.4) and the fact that the product $\|\nabla \alpha \otimes \nabla \alpha\|_{0,2}=\|\nabla \alpha\|_{0,4}^{2}$ is bounded we have

$$
\begin{aligned}
\mathcal{I}_{\mathrm{vK}}^{\theta}\left(u_{\theta}, v_{\theta}\right) & =\frac{1}{2} \int_{\omega} \int_{-1 / 2}^{1 / 2} Q_{2}\left(t, \theta^{1 / 2} A_{\theta}-t \nabla^{2} v_{\theta}+\check{B}(t)\right) \mathrm{d} t \mathrm{~d} x^{\prime} \\
& =\frac{1}{2} \int_{\omega} \int_{-1 / 2}^{1 / 2} Q_{2}\left(t, \nabla_{s} g+(\alpha-t) \nabla^{2} v+\check{B}(t)\right) \mathrm{d} t \mathrm{~d} x^{\prime}+\mathcal{O}\left(\theta^{-1 / 2}\right) .
\end{aligned}
$$

Now subtract and add $A_{\text {min }}$ inside $Q_{2}$ and use Cauchy's inequality to get

$$
\begin{aligned}
& \int_{-1 / 2}^{1 / 2} Q_{2}\left(t, \nabla_{s} g+\alpha \nabla^{2} v-t \nabla^{2} v+\check{B}\right) \mathrm{d} t \\
& \leqslant(1+\sqrt{\delta}) \int_{-1 / 2}^{1 / 2} Q_{2}\left(t, A_{\min }-t \nabla^{2} v+\check{B}\right) \mathrm{d} t \\
& \quad+\frac{1}{4 \sqrt{\delta}} \underbrace{\int_{-1 / 2}^{1 / 2} Q_{2}\left(t, \nabla_{s} g+\alpha \nabla^{2} v-A_{\min }\right) \mathrm{d} t}_{\lesssim\left\|\nabla_{s} g+\alpha \nabla^{2} v-A_{\min }\right\|_{0,2}^{2}<\delta} \\
& =\int_{-1 / 2}^{1 / 2} Q_{2}\left(t, A_{\min }-t \nabla^{2} v+\check{B}\right) \mathrm{d} t+\mathcal{O}_{\delta \downarrow 0}\left(\delta^{1 / 2}\right) .
\end{aligned}
$$

We plug this in and obtain:

$$
\begin{aligned}
\mathcal{I}_{\mathrm{vK}}^{\theta}\left(u_{\theta}, v_{\theta}\right) \leqslant & \frac{1}{2} \int_{\omega} \int_{-1 / 2}^{1 / 2} Q_{2}\left(t, A_{\min }-t \nabla^{2} v+\check{B}(t)\right) \mathrm{d} t \mathrm{~d} x^{\prime} \\
& +\mathcal{O}\left(\theta^{-1 / 2}\right)+\mathcal{O}_{\delta \downarrow 0}\left(\delta^{1 / 2}\right) \\
\stackrel{\theta \uparrow \infty}{\longrightarrow} & \frac{1}{2} \int_{\omega} \int_{-1 / 2}^{1 / 2} Q_{2}\left(t, A_{\min }-t \nabla^{2} v+\check{B}(t)\right) \mathrm{d} t \mathrm{~d} x^{\prime}+\mathcal{O}_{\delta \downarrow 0}\left(\delta^{1 / 2}\right) .
\end{aligned}
$$

The proof is concluded by letting $\delta \rightarrow 0$ and passing to a diagonal sequence. 
We finish the proof of Theorem 3.3 with the lower and upper bounds for the transition from $\alpha=3$ to $\alpha>3$. The lack of constraints in the limit functional makes the proofs straightforward.

Theorem 6.6 (Lower bound, von Kármán to linearised von Kármán). Let $\left(u_{\theta}, v_{\theta}\right)_{\theta>0}$ be a sequence in $X_{w}$ such that $\left(\theta^{1 / 2} u_{\theta}, v_{\theta}\right) \rightarrow(u, v)$ in $X_{w}$ as $\theta \rightarrow 0$. Then

$$
\operatorname{linf}_{\theta \rightarrow 0} \mathcal{I}_{\mathrm{vK}}^{\theta}\left(u_{\theta}, v_{\theta}\right) \geqslant \mathcal{I}_{\mathrm{lvK}}(u, v)
$$

Proof. We may assume that $\sup _{\theta>0} \mathcal{I}_{\mathrm{vK}}^{\theta}\left(u_{\theta}, v_{\theta}\right) \leqslant C$. Then by Theorem $6.3\left(\nabla v_{\theta}\right)_{\theta>0}$ is bounded in $W^{1,2}$ and by the Sobolev embedding $W^{1,2} \hookrightarrow L^{4}$ we have as before $\left\|\nabla v_{\theta} \otimes \nabla v_{\theta}\right\|_{0,2}=\left\|\nabla v_{\theta}\right\|_{0,4}^{2} \leqslant C$. Consequently

$$
\sqrt{\theta} A_{\theta}=\sqrt{\theta} \nabla_{s} u_{\theta}+\frac{\sqrt{\theta}}{2} \nabla v_{\theta} \otimes \nabla v_{\theta} \rightarrow \nabla_{s} u \quad \text { in } L^{2} \text { as } \theta \downarrow 0 .
$$

By convexity of the quadratic form $Q_{2}$ we conclude

$$
\begin{aligned}
\operatorname{linf}_{\theta \downarrow 0} \mathcal{I}_{\mathrm{vK}}^{\theta}\left(u_{\theta}, v_{\theta}\right) & \geqslant \frac{1}{2} \int_{\omega} \int_{-1 / 2}^{1 / 2} Q_{2}\left(x_{3}, \nabla_{s} u-x_{3} \nabla^{2} v+\check{B}\left(x_{3}\right)\right) \mathrm{d} x_{3} \mathrm{~d} x^{\prime} \\
& =\mathcal{I}_{\mathrm{lvK}}(u, v) .
\end{aligned}
$$

Theorem 6.7 (Upper bound, von Kármán to linearised von Kármán). Let $(u, v) \in X_{w}$. There exists a sequence $\left(u_{\theta}, v_{\theta}\right)_{\theta>0} \subset X_{w}$ such that $\left(\theta^{1 / 2} u_{\theta}, v_{\theta}\right) \rightarrow(u, v)$ in $X_{w}$ and $\mathcal{I}_{\mathrm{vK}}^{\theta}\left(u_{\theta}, v_{\theta}\right) \rightarrow \mathcal{I}_{\mathrm{lvK}}(u, v)$ as $\theta \rightarrow 0$.

Proof. Define

$$
u_{\theta}:=\theta^{-1 / 2} u \text { and } \quad v_{\theta}:=v
$$

Clearly $\left(\theta^{1 / 2} u_{\theta}, v_{\theta}\right) \equiv(u, v)$ and using again $W^{1,2} \hookrightarrow L^{4}$ we have:

$$
\sqrt{\theta} A_{\theta}=\nabla_{s} u+\frac{1}{2} \theta^{1 / 2} \nabla v \otimes \nabla v \underset{\theta \downarrow 0}{\longrightarrow} \nabla_{s} u \quad \text { in } L^{2}
$$

Consequently:

$$
\begin{aligned}
\mathcal{I}_{\mathrm{vK}}^{\theta}\left(u_{\theta}, v_{\theta}\right) & =\frac{1}{2} \int_{\omega} \int_{-1 / 2}^{1 / 2} Q_{2}\left(x_{3}, \sqrt{\theta} A_{\theta}-x_{3} \nabla^{2} v_{\theta}+\check{B}\left(x_{3}\right)\right) \mathrm{d} x_{3} \mathrm{~d} x^{\prime} \\
& \rightarrow \frac{1}{\theta \downarrow 0} \int_{\omega} \int_{-1 / 2}^{1 / 2} Q_{2}\left(x_{3}, \nabla_{s} u-x_{3} \nabla^{2} v+\check{B}\left(x_{3}\right)\right) \mathrm{d} x_{3} \mathrm{~d} x^{\prime} \\
& =\mathcal{I}_{\mathrm{lvK}}(u, v),
\end{aligned}
$$

as stated.

\section{Approximation And Representation theorems}

A key ingredient in the proofs of the upper bounds is the density of certain smooth functions in the space where the energy is minimised. In particular, for the case $\alpha \in(2,3)$ we obtain a result proving that $W^{2,2}$ maps with singular Hessian can be approximated by a specific set of smooth functions with the same property. In order to apply the results of [52] we may restrict ourselves to isometries which partition $\omega$ into finitely many 
so-called bodies and arms. More precisely, suppose $y: \omega \rightarrow \mathbb{R}^{3}$ is a $W^{2,2}$ isometric immersion and denote by $\mathrm{II}=\mathrm{II}_{(y)}$ its second fundamental form, i.e., $\mathrm{II}_{i j}=y_{, i} \cdot\left(y_{, 1} \wedge y_{, 2}\right)_{, j}$. Then II is singular, and there exists $f_{y} \in W^{1,2}$ such that $\nabla f_{y}=$ II. We call $\gamma:[0, l] \rightarrow \omega$, parameterised by arclength, a leading curve if it is orthogonal to the inverse images of $f_{y}$ on regions where $f_{y}$ is not constant. We denote by $\kappa$ and $\nu$ the curvature and unit normal, respectively, i.e., $\gamma^{\prime \prime}=\kappa \nu$. In fact, $\kappa$ must be bounded, hence $\gamma \in W^{2, \infty}$. A subdomain $\omega^{\prime} \subset \omega$ is said to be covered by a curve $\gamma$ if

$$
\omega^{\prime} \subset\{\gamma(t)+s \nu(t): s \in \mathbb{R}, t \in[0, l]\} .
$$

As shown in [46], if $\omega \subset \mathbb{R}^{2}$ is a bounded convex domain, it can be partitioned into so-called bodies and arms. Here a body is a connected maximal subdomain on which $y$ is affine and whose boundary contains more than two segments inside $\omega$. An arm is a maximal subdomain $\omega(\gamma)$ covered by some leading curve $\gamma$. Such a covering is possible also for general bounded Lipschitz domains, see ([26], Thms. 3 and 4).

In [52] (built on [46]) it is shown that for convex $\omega$ the set

$$
\mathcal{A}_{0}:=\left\{y \in C^{\infty}\left(\bar{\omega} ; \mathbb{R}^{3}\right): y \text { is an isometry finitely partitioning } \omega\right\}
$$

i.e., the set of $C^{\infty}$-smooth isometries with only a finite number of bodies and arms, is dense in the $W^{2,2}$ isometries. For Lipschitz domains, a direct application of the results in [26] only leads to approximations with possibly a countable number of arms within portions near the boundary. However, for domains $\omega$ for which there exists $\Sigma=\bar{\Sigma} \subset \partial \omega$ with $\mathcal{H}^{1}(\Sigma)=0$ such that on its complement $\partial \omega \backslash \Sigma$ the outer unit normal to $\omega$ exists and is continuous, $c f$. Remark 3.2.4, Hornung constructs in [25] $C^{\infty}$-smooth approximations to a given $W^{2,2}$-isometry, which are non-affine only on a finite number of arms. In this sense, the set $\mathcal{A}_{0}$ in (7.1) is still dense in the $W^{2,2}$-isometries if it is understood as the set of $C^{\infty}$-smooth isometries $y$ with only a finite number of bodies and arms on which $y$ is non-affine.

Here we show that, additionally,

$$
\mathcal{V}_{0}:=\left\{v \in C^{\infty}(\bar{\omega}): \exists \eta>0 \text { s.t. } \eta v=y_{3} \text { for some } y \in \mathcal{A}_{0}\right\}
$$

is $W^{2,2}$-dense in $W_{s h}^{2,2} \cdot 15$

Theorem 7.1. Let $\omega \subset \mathbb{R}^{2}$ be a bounded, convex ${ }^{16}$ domain. Then the set $\mathcal{V}_{0}$ is $W^{2,2}$-dense in $W_{\text {sh }}^{2,2}(\omega)$. In particular $\operatorname{det} \nabla^{2} v=0$ for all $v \in \mathcal{V}_{0}$.

Proof. Step 1: Approximation. Let $v \in W_{s h}^{2,2}(\omega)$ and $\varepsilon>0$. By ([23], Thm. 10), we can find some $\tilde{v} \in W_{s h}^{2,2}(\omega) \cap$ $W^{1, \infty}\left(\overline{\omega) \text { s.t. }\|v-\tilde{v}\|_{2,2}<\varepsilon / 2}\right.$ and, for $\eta=\eta(\varepsilon)>0$ sufficiently small, $\|\nabla \eta \tilde{v}\|_{\infty}<1 / 2$. One can now apply ([23], Thm. 7) to construct an isometry $\tilde{y} \in W^{2,2}\left(\omega ; \mathbb{R}^{3}\right)$ whose out-of-plane component $\tilde{y}_{3}=\eta \tilde{v}$. By the density of $\mathcal{A}_{0}$ we find a smooth $y \in \mathcal{A}_{0}$ such that $\|y-\tilde{y}\|_{2,2}<\varepsilon \eta / 2$ and in particular $\left\|y_{3}-\tilde{y}_{3}\right\|_{2,2}<\varepsilon \eta / 2$. Setting $\psi:=y_{3} / \eta \in \mathcal{V}_{0}$ we conclude

$$
\|v-\psi\|_{2,2} \leqslant\|v-\tilde{v}\|_{2,2}+\|\tilde{v}-\psi\|_{2,2}<\varepsilon
$$

\footnotetext{
${ }^{15}$ The density of $C^{2}(\omega) \cap W_{s h}^{2,2}(\omega)$ in $W_{s h}^{2,2}(\omega)$ was first announced in [46] to follow along the same lines as the density of smooth isometric immersions in the class of $W^{2,2}$ isometric immersions. As this seems not to be straightforward, we follow a different route reducing the density of $\mathcal{V}_{0}$ in $W_{s h}^{2,2}$ to the density of $\mathcal{A}_{0}$ in the set of $W^{2,2}$ isometric immersions. We are grateful to Peter Hornung for the help provided with this proof.

${ }^{16}$ Again the assumption of convexity may be dropped if one requires that $\omega$ still be simply connected and $\partial \omega$ satisfy the assumption detailed in Remark 3.2.4, because $\mathcal{A}_{0}$ is still dense in the set of $W^{2,2}$ isometric immersions and ([23], Thm. 7) holds true also in this situation.
} 
Step 2: Inclusion. Let $v \in \mathcal{V}_{0}$ with $\eta v=y_{3}, \eta>0$ for some smooth isometry $y \in \mathcal{A}_{0}$. Recall that the second fundamental form $\mathrm{II}_{(y)}$ of any smooth isometric immersion $y$ is singular and the identity $\nabla^{2} y_{j}=-\mathrm{II}_{(y)} n_{j}$ holds for all $j \in\{1,2,3\}$, where $n=y_{, 1} \wedge y_{, 2} \cdot{ }^{17}$ Therefore $\operatorname{det}\left(\eta \nabla^{2} v\right)=\operatorname{det}\left(-\mathrm{II}_{(y)} n_{3}\right)=0$ and the proof is complete.

As it appears to be of independent interest we state here the following condensed version of our previous considerations.

Remark 7.2. Let $\omega \subset \mathbb{R}^{2}$ be a bounded, simply connected, Lipschitz domain whose boundary contains a set $\Sigma=\bar{\Sigma} \subset \partial \omega$ with $\mathcal{H}^{1}(\Sigma)=0$ such that on its complement $\partial \omega \backslash \Sigma$ the outer unit normal to $\omega$ exists and is continuous. Then the set $W_{s h}^{2,2}(\omega) \cap C^{\infty}(\bar{\omega})$ is $W^{2,2}$-dense in $W_{s h}^{2,2}(\omega)$.

Once one can work with smooth functions, the essential tool for the construction of the recovery sequences for $\alpha \in(2,3)$ is the following representation theorem for maps with singular Hessian and its corollary, both inspired by [52]: In ([52], Lem. 3.3) it was shown that if $y \in \mathcal{A}_{0}$ and $A \in C^{\infty}\left(\bar{\omega} ; \mathbb{R}_{\mathrm{sym}}^{2 \times 2}\right)$ vanishes over a neighbourhood of $N=\left\{\mathrm{II}_{(y)}=0\right\}$, then there exist $\tilde{\alpha}, g_{1}, g_{2} \in C^{\infty}(\bar{\omega})$ vanishing on $N$ such that $A=\nabla_{s} g+\tilde{\alpha} \mathrm{II}_{(y)}$. While $\omega$ was assumed to be convex in [52], the same proof applies to the situation of domains $\omega$ where $\partial \omega$ satisfies the assumption detailed in Remark 3.2.4. This is because $\tilde{\alpha}, g_{1}, g_{2}$ are chosen to vanish on bodies and those arms where $y$ is affine and the construction in ([52], Lem. 3.3) is done over the finitely many covered domains over which $y$ is non-affine only.

Theorem 7.3. Let $\omega \subset \mathbb{R}^{2}$ be a bounded convex ${ }^{18}$ domain and $v \in \mathcal{V}_{0}$ and $A \in C^{\infty}\left(\bar{\omega} ; \mathbb{R}_{\mathrm{sym}}^{2 \times 2}\right)$ such that $A \equiv 0$ in a neighbourhood of $\left\{\nabla^{2} v=0\right\}$. There exist maps $\alpha, g_{1}, g_{2} \in C^{\infty}(\bar{\omega})$ such that $\alpha=g_{i}=0$ on $\left\{\nabla^{2} v=0\right\}$ and

$$
A=\nabla_{s} g+\alpha \nabla^{2} v
$$

Proof. Let $\eta>0, y \in \mathcal{A}_{0}$ s.t. $\eta v=y_{3}$. Using that $\nabla^{2} y_{3}=-\mathrm{II}_{(y)} n_{3}$ holds by virtue of $y$ being an isometry, with $n=y_{, 1} \wedge y_{, 2}$ being the unit normal vector, we have that $A \equiv 0$ in a neighbourhood of $\left\{\mathrm{II}_{(y)}=0\right\} \cup\left\{n_{3}=0\right\}$, and

$$
\left\{\nabla^{2} v=0\right\}=\left\{\mathrm{II}_{(y)}=0\right\} \cup\left\{n_{3}=0\right\} .
$$

We can apply the above stated ([52], Lem. 3.3) to $y$ in order to obtain functions $\tilde{\alpha}, g_{1}, g_{2} \in C^{\infty}(\bar{\omega})$ s.t. $\tilde{\alpha}, g_{1}, g_{2}=0$ on $\left\{\mathrm{II}_{(y)}=0\right\}$ and $A=\nabla_{s} g+\tilde{\alpha} \mathrm{II}_{(y)}$.

By examining the proof of this Lemma one can see that $\tilde{\alpha}, g \equiv 0$ in a neighbourhood of $\left\{n_{3}=0\right\}$ : since over bodies one has $\tilde{\alpha}, g_{1}, g_{2}=0$ by construction, we need only consider arms. On these sets, if $n_{3}$ vanishes at a point then it vanishes at a whole line perpendicular to the leading curve, because the latter is orthogonal to the level sets of the gradient. Now, because $A=0$ in a neighbourhood of this line, when solving the equations in the proof of the Lemma which determine $g$ then $\tilde{\alpha}$, one obtains $u_{2, s}=0$ and $u_{2, t}=0$, and with the boundary conditions $u_{2}=0$ then $u_{1}=0$ is a solution to the remaining equation. Hence $g=0$ and $\tilde{\alpha}=0$ on these lines. Since the functions so obtained are $C^{\infty}$, we can define $\alpha:=-\tilde{\alpha} \eta / n_{3}$ if $n_{3} \neq 0$ and $\alpha=0$ otherwise, and this is a smooth function such that

$$
A=\nabla_{s} g+\alpha \nabla^{2} v
$$

\footnotetext{
${ }^{17}$ See ([42], Prop. 3) for a proof for $W^{2,2}$ isometries on Lipschitz domains.

${ }^{18}$ Again instead $\omega$ being convex one may assume that it be simply connected and $\partial \omega$ satisfy the assumption detailed in Remark 3.2.4. Theorem 7.1, Corollary 7.4, ([23], Thm. 7) and ([52], Lem. 3.3) (and its proof) are valid in this situation as well.
} 
Corollary 7.4. Let $\omega$ be as in Theorem 7.3, $v \in \mathcal{V}_{0}$ and define for every $x^{\prime} \in \omega$

$$
A_{\min }\left(x^{\prime}\right)=\underset{A \in \mathbb{R}_{\text {sym }}^{2 \times 2}}{\operatorname{argmin}} \int_{-1 / 2}^{1 / 2} Q_{2}\left(t, A-t \nabla^{2} v\left(x^{\prime}\right)+\check{B}_{s}\right) \mathrm{d} t .
$$

Then $A_{\min } \in L^{2}\left(\omega ; \mathbb{R}_{\mathrm{sym}}^{2 \times 2}\right)$ and there exist sequences of functions $\alpha_{k} \in C^{\infty}(\bar{\omega}), g_{k} \in C^{\infty}\left(\bar{\omega} ; \mathbb{R}^{2}\right)$ such that

$$
\left\|\nabla_{s} g_{k}+\alpha_{k} \nabla^{2} v-A_{\min }\right\|_{L^{2}\left(\omega ; \mathbb{R}^{2 \times 2}\right)} \longrightarrow 0 \text { as } k \rightarrow \infty .
$$

Proof. Let $k \in \mathbb{N}$ be arbitrary. First, on the set $\left\{\nabla^{2} v=0\right\}$ we trivially have $A_{\min } \equiv A_{0}$ a constant matrix. Now let $A_{k} \in C^{\infty}\left(\bar{\omega} ; \mathbb{R}^{2 \times 2}\right)$ with support in $\left\{\nabla^{2} v \neq 0\right\}$ such that

$$
\left\|A_{k}-\left(A_{\min }-A_{0}\right)\right\|_{L^{2}\left(\omega ; \mathbb{R}^{2 \times 2}\right)}<\frac{1}{k}
$$

and use Theorem 7.3 to pick smooth $\alpha_{k}, \tilde{g}_{k}$ on $\bar{\omega}$ with

$$
A_{k}=\nabla_{s} \tilde{g}_{k}+\alpha_{k} \nabla^{2} v
$$

Set $g_{k}\left(x^{\prime}\right)=\tilde{g}_{k}\left(x^{\prime}\right)+A_{0} x^{\prime}$. Then:

$$
\left\|\nabla_{s} g_{k}+\alpha_{k} \nabla^{2} v-A_{\min }\right\|_{L^{2}}=\left\|\nabla_{s} \tilde{g}_{k}+\alpha_{k} \nabla^{2} v-\left(A_{\min }-A_{0}\right)\right\|_{L^{2}}<\frac{1}{k} .
$$

Acknowledgements. We are grateful to Peter Hornung for the help provided with the proof of Theorem 7.1. Also the valuable suggestions of the unknown referees are appreciated. This work was financially supported by project 285722765 of the Deutsche Forschungsgemeinschaft (DFG, German Research Foundation), "Effektive Theorien und Energie minimierende Konfigurationen für heterogene Schichten".

\section{REFERENCES}

[1] H. Abels, M.G. Mora and S. Müller, The time-dependent von Kármán plate equation as a limit of 3d nonlinear elasticity. Calc. Var. Partial Differ. Equ. 41 (2011) 241-259.

[2] E. Acerbi, G. Buttazzo and D. Percivale, Thin inclusions in linear elasticity: A variational approach. J. Reine Angew. Math. 386 (1988) 99-115.

[3] E. Acerbi, G. Buttazzo and D. Percivale, A variational definition of the strain energy for an elastic string. J. Elast. 25 (1991) $137-148$.

[4] G. Anzellotti, S. Baldo and D. Percivale, Dimension reduction in variational problems, asymptotic development in $\Gamma$-convergence and thin structures in elasticity. Asymptot. Anal. 9 (1994) 61-100.

[5] H. B. Belgacem, S. Conti, A. DeSimone and S. Müller, Energy scaling of compressed elastic films - three-dimensional elasticity and reduced theories. Arch. Ration. Mech. Anal. 164 (2002) 1-37.

[6] K. Bhattacharya, M. Lewicka and M. Schäffner, Plates with incompatible prestrain. Arch. Ration. Mech. Anal. 221 (2016) $143-181$.

[7] A. Braides, A handbook of $\Gamma$-convergence, in Stationary Partial Differential Equations, edited by M. Chipot and P. Quittner, Vol. 3. Handbook of Differential Equations. Elsevier (2006) 101-213.

[8] J. Braun and B. Schmidt, An atomistic derivation of von-Kármán plate theory. Preprint https://arxiv.org/abs/1907.00197 (2019).

[9] P.G. Ciarlet, Mathematical Elasticity. Vol. II: Theory of Plates, Vol. 27. Studies in Mathematics and Its Applications. North-Holland Publishing Co., Amsterdam (1997).

[10] P.G. Ciarlet, Mathematical Elasticity. Vol. III: Theory of Shells, Vol. 29. Studies in Mathematics and Its Applications. North-Holland Publishing Co., Amsterdam (2000).

[11] S. Conti, Low-Energy Deformations of Thin Elastic Plates: Isometric Embeddings and Branching Patterns. Habilitationsschreiben, Universität Leipzig (2004).

[12] S. Conti and G. Dolzmann, Г-convergence for incompressible elastic plates. Calc. Var. Partial Diff. Equ. 34 (2009) 531-551. 
[13] S. Conti and F. Maggi, Confining thin elastic sheets and folding paper. Arch. Ration. Mech. Anal. 187 (2008) 1-48.

[14] M. de Benito Delgado, Effective two dimensional theories for multi-layered plates. Doctoral dissertation, Universität Augsburg (2019).

[15] M. de Benito Delgado and B. Schmidt, Energy minimizing configurations of pre-strained multilayers. J. Elast. 140 (2020) 303-335.

[16] E. Efrati, E. Sharon and R. Kupferman, Elastic theory of unconstrained non-Euclidean plates. J. Mech. Phys. Solids 57 (2009) $762-775$.

[17] A.I. Egunov, J.G. Korvink and V.A. Luchnikov, Polydimethylsiloxane bilayer films with an embedded spontaneous curvature. Soft Matter 12 (2016) 45-52.

[18] L. Euler, Methodus inveniendi lineas curvas, additamentum I: De curvis elasticis (1744), in Opera Omnia Ser. Prima, Vol. XXIV. Orell Füssli, Bern (1952) 231-297.

[19] M. Finot and S. Suresh, Small and large deformation of thick and thin-film multi-layers: Effects of layer geometry, plasticity and compositional gradients. Mechanics and Physics of Layered and Graded Materials. J. Mech. Phys. Solids 44 (1996) 683-721.

[20] L.B. Freund, Substrate curvature due to thin film mismatch strain in the nonlinear deformation range. The J. R. Willis 60 th anniversary volume. J. Mech. Phys. Solids 48 (2000) 1159-1174.

[21] G. Friesecke, R.D. James, M.G. Mora and S. Müller. Derivation of nonlinear bending theory for shells from three-dimensional nonlinear elasticity by Gamma-convergence. C. R. Math. Acad. Sci. Paris 336 (2003) 697-702.

[22] G. Friesecke, R.D. James and S. Müller, A theorem on geometric rigidity and the derivation of nonlinear plate theory from three-dimensional elasticity. Commun. Pure Appl. Math. 55 (2002) 1461-1506.

[23] G. Friesecke, R.D. James and S. Müller, A hierarchy of plate models derived from nonlinear elasticity by $\Gamma$-convergence. Arch. Ration. Mech. Anal. 180 (2006) 183-236.

[24] M. Grundmann, Nanoscroll formation from strained layer heterostructures. Appl. Phys. Lett. 83 (2003) $2444-2446$.

[25] P. Hornung, Approximation of flat $W^{2,2}$ isometric immersions by smooth ones. Arch. Ration. Mech. Anal. 199 (2011) $1015-1067$.

[26] P. Hornung, Fine level set structure of flat isometric immersions. Arch. Ration. Mech. Anal. 199 (2011) $943-1014$.

[27] P. Hornung, S. Neukamm, and I. Velčić, Derivation of a homogenized nonlinear plate theory from 3d elasticity. Calc. Var. Partial Diff. Equ. 51 (2014) 677-699.

[28] P. Hornung, M. Pawelczyk and I. Velčić, Stochastic homogenization of the bending plate model. J. Math. Anal. Appl. 458 (2018) 1236-1273.

[29] C.S. Kim and S.J. Lombardo, Curvature and bifurcation of $\mathrm{MgO}-\mathrm{Al}_{2} \mathrm{O}_{3}$ bilayer ceramic structures. J. Ceram. Process. Res. 9 (2008) 93-96.

[30] G. Kirchhoff, Über das Gleichgewicht und die Bewegung einer elastischen Scheibe. J. Reine Angew. Math. 40 (1850) 51-88.

[31] Y. Klein, E. Efrati and E. Sharon, Shaping of elastic sheets by prescription of non-euclidean metrics. Science 315 (2007) 1116-1120.

[32] R. Kupferman and J.P. Solomon, A Riemannian approach to reduced plate, shell, and rod theories. J. Funct. Anal. 266 (2014) 2989-3039.

[33] H. Le Dret and A. Raoult, The nonlinear membrane model as variational limit of nonlinear three-dimensional elasticity. $J$. Math. Pures Appl. 74 (1995) 549-578.

[34] M. Lewicka and D. Lučić, Dimension reduction for thin films with transversally varying prestrian: the oscillatory and the non-oscillatory case. Preprint https://arxiv.org/abs/1807.02060 (2018).

[35] M. Lewicka, L. Mahadevan and M.R. Pakzad, The Föppl-von Kármán equations for plates with incompatible strains. Proc. Roy. Soc. London Ser. A. Math. Phys. Eng. Sci. 467 (2011) 402-426.

[36] M. Lewicka, M.G. Mora and M.R. Pakzad, Shell theories arising as low energy $\Gamma$-limit of 3d nonlinear elasticity. Annali della Scuola normale superiore di Pisa, Classe di scienze 9 (2008) 253-295.

[37] A.E.H. Love, A Treatise on the Mathematical Theory of Elasticity, 4th edn. Dover Publications, New York (1944).

[38] C. Maor and A. Shachar, On the role of curvature in the elastic energy of non-Euclidean thin bodies. J. Elast. 134 (2019) $149-173$.

[39] C.B. Masters and N. Salamon, Geometrically nonlinear stress-deflection relations for thin film/substrate systems. Int. J. Eng. Sci. 31 (1993) 915-925.

[40] M.G. Mora, S. Müller and M.G. Schultz, Convergence of equilibria of planar thin elastic beams. Indiana Univ. Math. J. 56 (2007) 2413-2438

[41] S. Müller, Mathematical problems in thin elastic sheets: scaling limits, packing, crumpling and singularities, in Vector-Valued Partial Differential Equations and Applications, Vol. 2179. Lecture Notes Math. Springer, Cham (2017) 125-193.

[42] S. Müller and M.R. Pakzad, Regularity properties of isometric immersions. Mathematische Zeitschrift 251 (2005) 313-331.

[43] S. Müller and M.R. Pakzad, Convergence of equilibria of thin elastic plates - the von Kármán case. Comm. Partial Differ. Equ. 33 (2008) 1018-1032.

[44] S. Neukamm and I. Velčić, Derivation of a homogenized von-Kármán plate theory from 3D nonlinear elasticity. Math. Models Methods Appl. Sci. 23 (2013) 2701-2748.

[45] H. Paetzelt, V. Gottschalch, J. Bauer, H. Herrnberger and G. Wagner. Fabrication of III-V nano- and microtubes using MOVPE grown materials. Physica Status Solidi (A) 203 (2006) 817-824.

[46] M.R. Pakzad, On the Sobolev space of isometric immersions. J. Differ. Geom. 66 (2004) 47-69. 
[47] V.Y. Prinz, D. Grützmacher, A. Beyer, C. David, B. Ketterer and E. Deckardt, A new technique for fabricating threedimensional micro- and nanostructures of various shapes. Nanotechnology 12 (2001) 399-402.

[48] J.N. Reddy, Mechanics of Laminated Composite Plates and Shells: Theory and Analysis, 2nd edn. CRC Press (2003).

[49] N. Salamon and C.B. Masters, Bifurcation in isotropic thinfilm/substrate plates. Special topics in the theory of elastic: A volume in honour of Professor John Dundurs. Int. J. Solids Struct. 32 (1995) 473-481.

[50] B. Schmidt, A derivation of continuum nonlinear plate theory from atomistic models. Multiscale Model. Simul. 5 (2006) 664-694.

[51] B. Schmidt, Minimal energy configurations of strained multi-layers. Cal. Var. Partial Diff. Equ. 30 (2007) $477-497$.

[52] B. Schmidt, Plate theory for stressed heterogeneous multilayers of finite bending energy. J. Math. Pures Appl. 88 (2007) $107-122$.

[53] B. Schmidt, A Griffith-Euler-Bernoulli theory for thin brittle beams derived from nonlinear models in variational fracture mechanics. Math. Models Methods Appl. Sci. 27 (2017) 1685-1726.

[54] O.G. Schmidt and K. Eberl, Thin solid films roll up into nanotubes. Nature 410 (2001) 168.

[55] T. von Kármán, Festigkeitsprobleme im Maschinenbau, in Encyclopädie der Mathematischen Wissenschaften, Vol. IV/4. Teubner, Leipzig (1910) 311-385. 